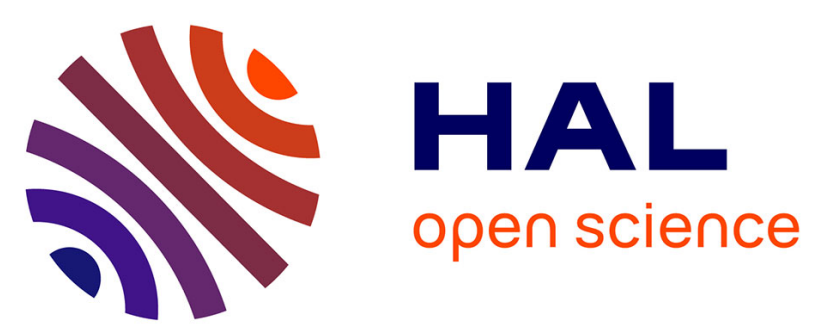

\title{
Scheduling analysis of tasks constrained by TDMA: Application to software radio protocols
}

\author{
Shuai Li, Frank Singhoff, Stéphane Rubini, Michel Bourdellès
}

\section{To cite this version:}

Shuai Li, Frank Singhoff, Stéphane Rubini, Michel Bourdellès. Scheduling analysis of tasks constrained by TDMA: Application to software radio protocols. Journal of Systems Architecture, 2017, 76, pp.5875. 10.1016/j.sysarc.2016.11.003 . hal-01685444

\section{HAL Id: hal-01685444 https://hal.univ-brest.fr/hal-01685444}

Submitted on 16 Jan 2018

HAL is a multi-disciplinary open access archive for the deposit and dissemination of scientific research documents, whether they are published or not. The documents may come from teaching and research institutions in France or abroad, or from public or private research centers.
L'archive ouverte pluridisciplinaire HAL, est destinée au dépôt et à la diffusion de documents scientifiques de niveau recherche, publiés ou non, émanant des établissements d'enseignement et de recherche français ou étrangers, des laboratoires publics ou privés. 


\title{
Scheduling Analysis of Tasks Constrained by TDMA: Application to Software Radio Protocols Author's version Shuai Li+*, Frank Singhoff*, Stéphane Rubini*, Michel Bourdellès+ *Lab-STICC UMR 6285, Université de Bretagne Occidentale, France, \{last-name\}@univ-brest.fr +Thales Communications \& Security, France, \{first-name\}.\{last-name\}@thalesgroup.com
}

July 20, 2017

\begin{abstract}
:
The work, presented in this article, aims at performing scheduling analysis of Time Division Multiple Access (TDMA) based communication systems. Products called software radio protocols, developed at Thales Communications \& Security, are used as case-studies.

Such systems are real-time embedded systems. They are implemented by tasks that are statically allocated on multiple processors. A task may have an execution time, a deadline and a release time that depend on TDMA configuration. The tasks also have dependencies through precedence and shared resources. TDMA-based software radio protocols have architecture characteristics that are not handled by scheduling analysis methods of the literature. A consequence is that existing methods give either optimistic or pessimistic analysis results.

We propose a task model called Dependent General Multiframe (DGMF) to capture the specificities of such a system for scheduling analysis. The DGMF task model describes, in particular, the different jobs of a task, and task dependencies. To analyze DGMF tasks, we propose their transformation to real-time transactions and we also propose a new schedulability test for such transactions.

The general analysis method is implemented in a tool that can be used by software engineers and architects. Experimental results show that our proposi-
\end{abstract}


tions give less pessimistic schedulability results, compared to fundamental methods. The results are less pessimistic for both randomly generated systems and real case-studies from Thales.

These results are important for engineering work, in order to limit the overdimensioning of resources. Through tooling, we also automated the analysis. These are advantages for engineers in the more and more competitive market of software radios.

Keywords: Real-time Embedded System, Scheduling Analysis, Task Dependency, Software Radio Protocol, TDMA, General Multiframe, Transaction

\section{Introduction}

In this article, we propose a scheduling analysis method for communication systems that use the Time Division Multiple Access (TDMA) channel access method. Our method is applied to real industrial systems, developed at Thales Communications \& Security, called software radio protocols.

\subsection{Context}

Radios are communication systems. For communication to be established, some radio protocols need to be defined and implemented. Traditionally radios are implemented as dedicated hardware components. Trends in the last two decades have seen the emergence of software radios [1], where more and more components are implemented as software. This is the case for the radio protocol, which is then called a software radio protocol.

Software radio protocols are typical real-time systems. In such systems, activities are implemented by software tasks that are time-constrained. The tasks may also have precedence dependencies and use shared resources. In this article, we consider tasks that are scheduled by a partitioned Fixed Priority (FP) preemptive scheduling policy.

The particularity of a software radio protocol real-time system is that it may be impacted by TDMA, a common method to access the shared communication medium between several radio stations. In TDMA 2, time is divided into several time slots and at each time slot, a radio either transmits its data, or receives some data. This method impacts the software of the system, because the tasks have an execution time, deadline, and release time that depend on the time slots. TDMA is also one reason, among others, that there are hard deadlines in such systems. We must thus analyze the scheduling of these systems.

Scheduling analysis [3, 4] is the method used to verify that tasks will meet their deadline, when they are scheduled on processors. Scheduling analysis can potentially be complex, due to the dependencies between the tasks for example. Some methods, part of scheduling analysis, are schedulability tests [5]. Such tests assesses if all jobs, of all tasks, will meet their deadlines during execution, when the tasks are scheduled on processors by a specific scheduling policy. Schedulability tests may compute the Worst Case Response Time (WCRT) of 
tasks. The WCRT of a task is compared to its deadline to assess its schedulability.

Schedulability tests are associated with task models that abstract the architecture of a real-time system for the analysis. There are several task models in the literature that consider more or less dependencies between tasks, and characteristics of the execution platform that hosts the tasks.

\subsection{Overview of Contributions}

As we will see, current task models and schedulability tests are not applicable to a software radio protocol that uses TDMA to access the shared communication medium. For example some task models may consider the impact of TDMA on tasks, but they do not consider task dependencies. Therefore, in this article we propose a scheduling analysis method adapted for the characteristics of real software radio systems developed at Thales. Our analysis method is based on several of our contributions. The next paragraphs describe our contributions and give an overview of our approach.

Extension of the General Multiframe Task Model This article proposes the Dependent General Multiframe (DGMF) task model, an extension of the General Multiframe (GMF) task model [6. A GMF task is a vector of frames that represent the jobs of the task. The jobs may not have the same parameters, such as deadline and execution time. The DGMF task model extends GMF with precedence dependencies and shared resources. Contrary to GMF, the DGMF task model can also be applicable to a multiprocessor system with partitioned scheduling.

DGMF to Transaction The analysis method for DGMF consists in exploiting another kind of model called transactions [7. A transaction is a group of tasks related by precedence dependency. This article proposes an algorithm to transform DGMF tasks to transactions. The transformation solves the issue of the semantic gap between the two models.

Extension of Schedulability Test for Transactions The transactions that are the results of the transformation have what we call non-immediate tasks, i.e. tasks which are not necessarily released immediately by their predecessor. Thus this article proposes the WCDSOP+NIM test, which is an extension of the WCDOPS + test in [8. Our test is used to assess schedulability of transactions with non-immediate tasks. Thanks to WCDSOP+NIM, we are able to perform scheduling analysis on transactions produced from DGMF models, and thus to perform scheduling analysis on DGMF models themselves.

Available Tool The GMF, DGMF, transaction models, and their analysis methods, are all implemented in Cheddar [9]. Cheddar is an open-source 


\begin{tabular}{|c|c|c|c|c|c|c|}
\hline \multicolumn{7}{|c|}{ TDMA Frame } \\
\hline S & B & B & $\mathbf{T}$ & $\mathbf{T}$ & $\mathbf{T}$ & $\mathbf{T}$ \\
\hline
\end{tabular}

Figure 1: TDMA Frame

scheduling analysis tool, available for both the research and industrial communities.

\subsection{Article Organization}

The rest of this article is divided into five sections that are organized as follows. In Section 2, the TDMA-based software radio protocol system is presented. This section defines the assumptions of our work. In Section 3, we discuss the applicability of task models related to our work. In Section 4 the DGMF task model, and its analysis method, and experiments (both simulation results and case-study results) are exposed. Section 5 is on our WCDOPS+NIM schedulability test. Again, experiments on our test are shown in the same section, with both simulation results and case-study results. Finally, we conclude in Section 6 by discussing some future works.

\section{Software Radio Protocol}

In the following sections, we first present the concept of a radio protocol and the impact of TDMA on the protocol. Afterwards the software and execution platform of our system is presented, and we relate the architecture of the system to two typical architecture designs. Finally, we summarize the requirements to consider for our scheduling analysis work.

\subsection{Radio Protocols Based On Time-Division Multiplex- ing}

A radio protocol is a set of rules to respect so communication can be established between different users on a same network. A radio protocol defines the syntax and semantics of the messages exchanged between users, but also how communication is synchronized between all users. Several protocols can cooperate within a same radio to form the protocol stack [10].

The functionalities of a radio protocol may be impacted by the method used by the radio to access the communication medium. TDMA [2] is a channel access method, based on time-division multiplexing. It allows several radio stations to transmit over a same communication medium. In TDMA, time is divided into several time slots, called TDMA slots. At each slot, each radio station in the network either transmits or receives. The sequence of slots is represented as a TDMA frame. Figure 1 shows a typical frame. 
Slots may be of different types. For example in Figure 1, the TDMA frame has three types of slot: Service $(S)$ for synchronization between stations; Beacon $(B)$ for observation/signaling the network; Traffic $(T)$ for payload transmission/reception. Slots of different types do not necessarily have the same characteristics. One of the characteristics of a slot is its duration. Therefore slots of different types do not necessarily have the same duration. For example in Figure 1 a $B$ slot duration is shorter than a $S$ and $T$ slot duration. The TDMA frame duration is the sum of durations of its slots. Slots can either be in transmission $(T x)$, reception $(R x)$, or Idle mode. In a $T x$ slot, a radio station can thus transmit data. When a radio station can transmit in a slot, we say that the slot is allocated to the station. A TDMA configuration defines the combination of slots, of different types, in a TDMA frame. A TDMA frame is repeated after it finishes. An instance of a frame is a cycle.

When TDMA is used to access the communication medium, it impacts the functionalities of the radio protocol and it introduces time constraints. For example the operation to fetch data packets to be transmitted in a $T x$ slot is time-constrained. The TDMA method is a particular case of the timedtoken protocol for real-time communications [11. Indeed the same approach of dividing time into time slots, and allocating slots to entities, is used in both methods. Since these methods introduce time constraints, tasks that implement the system may also have time constraints.

The next section exposes the software and execution platform architecture of one possible implementation of a radio protocol.

\subsection{Software and Execution Platform Architecture}

The previous section showed how a radio protocol is designed. This section presents one possible implementation by Thales, called a software radio protocol [1]. The implementation is described through its software and execution platform architecture.

Traditionally functionalities of a radio protocol are implemented as dedicated hardware (e.g. ASIC, FPGA). There is no concurrency to access these computing resources. Scheduling analysis is thus not necessary. In the case of systems developed at Thales, the majority of the functionalities of the protocol is implemented as software running on a General Purpose Processor (GPP). The software entities are implemented by some entities in the execution platform: OS and hardware. Figure 2 shows an example of these entities.

Figure 2, shows several protocols (called RLC and MAC) implemented by tasks allocated on processors. The system is a partitioned multiprocessor system: tasks are allocated on a processor, and they do not migrate. On a processor, tasks are scheduled by a preemptive FP scheduling policy. The priorities of tasks are assigned arbitrarily according to domain expertise.

They may have precedence dependency (e.g. communication through semaphores signaling). They may also use shared resources. Shared resources are assumed local [12, i.e. two tasks can use a shared resource only if they are allocated on the same processor. Shared resources are protected by a resource access proto- 


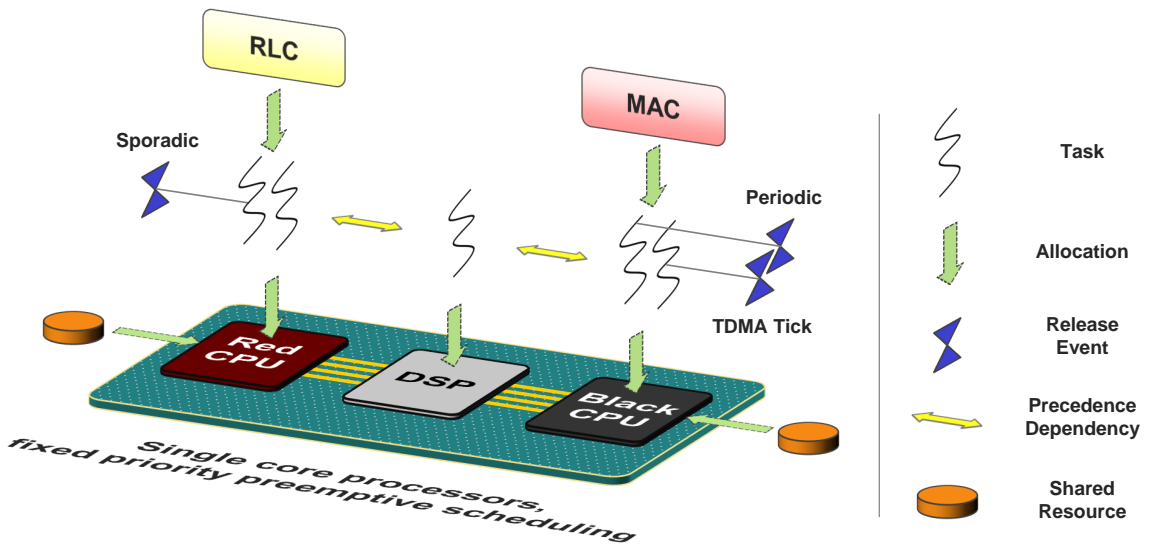

Figure 2: Architecture for Scheduling Analysis: Red CPU is a GPP handling ciphered data, Black CPU is a GPP handling deciphered data, DSP means Digital Signal Processor

col that prevents unbounded priority inversion for uniprocessor. Examples of such resource access protocols are PIP and PCP [13. Their implementation can typically be found in execution platforms with the VxWorks OS. This OS is present in some products developed by Thales.

Some tasks may be released sporadically. For example some tasks are typically released upon arrival of IP packets, which depends on the user application. Other tasks are released at pre-defined times. This is the case for tasks of a Medium Access Control (MAC) protocol [10. In this article, activities of MAC are divided in time due to the usage of TDMA to access the communication medium. The releases of a task can follow a periodic pattern or a pattern defined by TDMA slots. Events called TDMA ticks indicate the start of a slot and thus the release of some tasks. Tasks that are released at the start of a slot may also have execution times and deadlines that are constrained by the slot.

The software architecture of the system is conform to some architectures found in the literature. Indeed, two software architectures for RTES have been studied extensively: event-triggered [14] and time-triggered [15]. A software radio protocol is both a time-triggered and an event-triggered system. Indeed, some tasks are released by TDMA ticks indicating the start of a slot. Thus certain tasks are released at pre-defined times, which is conform to the timetriggered architecture. On the other hand, there are also tasks released by sporadic events, for example upon arrival of IP packets. Due to precedence dependency, a task can also be released by an event produced by a predecessor task. 


\subsection{Requirements for Scheduling Analysis}

From the description of its architecture in Section 2.2, characteristics of a software radio protocol, useful for scheduling analysis, are summarized in Table 1. In this article, it is expected that the proposed scheduling analysis method covers all of the characteristics in Table 1 .

In the next section we study the applicability of scheduling analysis methods of the literature, when considering the requirements exposed in this section.

\section{Applicability of Related Works}

In this section, we discuss problems encountered when applying existing task models of the literature, to analyze the scheduling of a software radio protocol.

A task model is said applicable to a characteristic of the system, if it is possible to model the characteristic, and if there exists a schedulability test for the task model that considers the characteristic. Table 2 shows the applicability of some task models to each characteristic. These task models come from the literature and they are the fundamental periodic task model [3], the transaction model [7] that expresses precedence dependent tasks, the multiframe task models [6] that express jobs of a task as a vector, and the DAG task models [5] that express jobs of a task as a graph.

The applicability of the task models is discussed in the following paragraphs. Either the non-applicability of some task models on a characteristic is justified, or the applicability of all task models on a characteristic is shown.

TDMA release Seminal schedulability tests in [3, 16, for the fundamental periodic and sporadic task models assume a synchronous system, where tasks are all released at a unique critical instant. Tasks constrained by TDMA are not synchronous since they may be released at different pre-defined times.

The transaction model, that generalizes the fundamental periodic and sporadic task models, focuses on asynchronous releases of tasks. Tasks are said asynchronous if there is at least one first job of a task that is not released at the same time as the other first jobs of other tasks. In [7, Tindell analyzes asynchronous tasks with the transaction model. He takes the example of a network with a bus and messages scheduled by TDMA. His goal was not to analyze the effect that TDMA has on the scheduling of tasks within a single system (e.g. job execution time and release time constrained by the TDMA slot), but endto-end response times of message transiting in the whole network. For example Tindell computes the response time from the sending of a message by a task, to the transition of the message on the bus, to the reception by another task.

Some other schedulability tests for transactions in [17, 8] also consider that there are some periodic tasks, and some tasks released immediately by their predecessor, instead of pre-defined times. These results cannot be applied on the targeted systems of this article. 
Table 1: Requirements for Scheduling Analysis

\begin{tabular}{|c|c|}
\hline Name & Description \\
\hline TDMA release & $\begin{array}{l}\text { Tasks may be released at pre- } \\
\text { defined times, corresponding to } \\
\text { the start of TDMA slots, accord- } \\
\text { ing to the TDMA configuration }\end{array}$ \\
\hline Periodic and sporadic release & $\begin{array}{l}\text { Tasks may be released by peri- } \\
\text { odic and sporadic events. }\end{array}$ \\
\hline Arbitrary deadline & Deadlines are arbitrarily defined. \\
\hline Arbitrary priority & $\begin{array}{l}\text { In case of FP scheduling, priori- } \\
\text { ties are arbitrarily defined. }\end{array}$ \\
\hline Precedence dependency & $\begin{array}{l}\text { Two tasks may have a prece- } \\
\text { dence dependency. }\end{array}$ \\
\hline Local Shared resource & $\begin{array}{l}\text { Tasks may use local shared re- } \\
\text { sources and thus have critical } \\
\text { sections. Shared resources are } \\
\text { protected by an access protocol } \\
\text { that prevents deadlocks and un- } \\
\text { bounded priority inversion time. }\end{array}$ \\
\hline Individual job parameter & $\begin{array}{l}\text { Task execution time and dead- } \\
\text { line may be constrained by the } \\
\text { nature of the release event (e.g. } \\
\text { TDMA slot), and may be dif- } \\
\text { ferent from one job to the } \\
\text { other. Precedence dependency } \\
\text { and shared resource critical sec- } \\
\text { tions may also be different from } \\
\text { one job to the other. }\end{array}$ \\
\hline Preemptive FP policy on uniprocessor & $\begin{array}{l}\text { On a uniprocessor, tasks are } \\
\text { scheduled according to a preemp- } \\
\text { tive FP scheduling policy. }\end{array}$ \\
\hline Partitioned multiprocessor & $\begin{array}{l}\text { A task is allocated on a proces- } \\
\text { sor and does not migrate. Tasks } \\
\text { on different processors may com- } \\
\text { municate. }\end{array}$ \\
\hline
\end{tabular}


Table 2: Applicability of Task Models to Software Radio Protocol: Abbreviations are $\mathrm{P} / \mathrm{S}=$ Periodic/Sporadic, $\mathrm{TR}=$ Transactions, $\mathrm{GMF}=$ Multiframe Task Models, DAG $=$ DAG Task Models; $\mathrm{N}=$ No, $\mathrm{Y}=$ Yes

\begin{tabular}{|l|c|c|c|c|}
\hline & $\mathrm{P} / \mathrm{S}$ & $\mathrm{TR}$ & $\mathrm{GMF}$ & $\mathrm{DAG}$ \\
\hline TDMA release & $\mathrm{N}$ & $\mathrm{N}$ & $\mathrm{Y}$ & $\mathrm{Y}$ \\
\hline Periodic and sporadic release & $\mathrm{Y}$ & $\mathrm{Y}$ & $\mathrm{Y}$ & $\mathrm{Y}$ \\
\hline Arbitrary deadline & $\mathrm{N}$ & $\mathrm{Y}$ & $\mathrm{N}$ & $\mathrm{Y}$ \\
\hline Arbitrary priority & $\mathrm{Y}$ & $\mathrm{Y}$ & $\mathrm{Y}$ & $\mathrm{Y}$ \\
\hline Precedence dependency & $\mathrm{N}$ & $\mathrm{Y}$ & $\mathrm{N}$ & $\mathrm{N}$ \\
\hline Local shared resource & $\mathrm{Y}$ & $\mathrm{Y}$ & $\mathrm{N}$ & $\mathrm{N}$ \\
\hline Individual job parameter & $\mathrm{N}$ & $\mathrm{N}$ & $\mathrm{Y}$ & $\mathrm{Y}$ \\
\hline Preemptive FP policy on uniprocessor & $\mathrm{Y}$ & $\mathrm{Y}$ & $\mathrm{Y}$ & $\mathrm{Y}$ \\
\hline Partitioned multiprocessor & $\mathrm{N}$ & $\mathrm{Y}$ & $\mathrm{N}$ & $\mathrm{N}$ \\
\hline
\end{tabular}

Finally some works have been done on systems that are both event-triggered and time-triggered, like a software radio protocol. In [18, the authors work on an automotive system respecting the design of both architectures. Their work does not focus on task scheduling, but network scheduling. Indeed, their objective is to optimize the TDMA slot duration, when a bus is accessed by several processors through the TDMA channel access method.

Periodic and sporadic release All task models support tasks released by a periodic or a sporadic event [3, 7, 6, 19].

Arbitrary deadline Some of the schedulability tests for the periodic and sporadic task models assume $D_{i} \leq T_{i}[3,16$. Thus the deadline is constrained.

Schedulability tests [6, 20] for GMF assume that the l-MAD property or the Frame Separation property hold. The l-MAD property constrains the deadline of a frame to be less than, or equal to, the deadline of the next frame. The Frame Separation property constrains the deadline of a frame to be less than the release of the next frame. These properties thus constrain the deadline of frames and thus tasks.

Arbitrary priority There exists at least one schedulability test for each task model that supports this characteristic [16, 21, 20, 5].

Precedence dependency Analysis of the periodic and sporadic task models did not fully handle precedence dependencies, until they were generalized by the transaction model [21.

Some works 22, 23, 7, 24, propose a method to schedule precedence dependent periodic or sporadic tasks. These methods either constrain the fixed priorities of tasks, or their deadlines, or they are proposed for a Earliest-Deadline First (EDF) policy with dynamic priorities instead of fixed one. Therefore they 
are not applicable due to the arbitrary priorities, arbitrary deadlines, and FP scheduling policy of a software radio protocol.

Other works [25] also focus on periodic tasks with precedence dependencies that are not specified for every job, but every $2,3, \ldots, n$ jobs. These works are not applicable to a software radio protocol, because precedence dependencies, among task jobs, do not follow this kind of pattern.

The multiframe task models assume independent tasks [6]. The DAG task models support precedence dependencies between jobs of a task. They do not explicitly handle precedence dependencies between the tasks themselves. For example the authors in [26] assume independent DAG tasks.

Local shared resource Like precedence dependencies, shared resources are not supported by the classical multiframe task models and DAG task models, since they assume independent tasks [6, 26. In [27 the authors propose an optimal shared resource access protocol for GMF tasks but the protocol is not implemented in the OS of Thales products.

Individual job parameter The modeling of jobs with different parameters is only supported by the multiframe and DAG task models [6, 5, 26, among those present in Table 2.

Preemptive FP policy on uniprocessor All task models have schedulability tests for the preemptive FP scheduling policy [3, 7, 6, 26].

Partitioned multiprocessor The periodic, sporadic task models, and the multiframe task models have schedulability tests for uniprocessor systems 3, 6]. The DAG task models has tests for uniprocessor systems [5], or global multiprocessor systems [26, i.e. where tasks may migrate to other processors. Again, these task models are not applicable to our system.

In conclusion, the results of Table 2 show that none of the task models support all characteristics of the software radio protocol to analyze. The following two sections expose our contributions to solve this problem: the DGMF task model, its transformation to a transaction model, and our extended schedulability test for transactions.

\section{Dependent General Multiframe}

To analyze the schedulability of a TDMA-based software radio protocol, this article proposes the Dependent General Multiframe (DGMF) task model. The following sections first define this task model. Then its analysis method is proposed. Finally some experimental results and evaluation are exposed. 


\subsection{DGMF, an Extension of GMF}

Jobs with individual parameters is frequent in the multimedia domain. For example a video decoder task of a multimedia system has a sequence of images to decode. The parameters of a job of the task are constrained by the type of the image to decode. The described task behavior also occurs in software radio protocols. Indeed a job of a task, released by TDMA slots, may also have parameters constrained by the slot. The job parameters are thus constrained by the sequence of slots of the TDMA frame.

A task model motivated by the described behavior is the GMF task model. As a reminder, a GMF task is an ordered vector of frames representing its jobs. Each frame can have a different execution time, deadline, and minimum separation time from the frame's release to the next frame's release. Among task models that propose to model individual job parameters, GMF is sufficient for the modeling of a sequence of task jobs, constrained by a sequence of TDMA slots. On the other hand, GMF is limited to uniprocessor systems, without task dependencies, and with constrained deadlines. The GMF task model is thus extended to propose a task model called DGMF.

The DGMF task model extends the GMF model with task dependencies. It is also applicable to partitioned multiprocessor systems. The following sections first define the DGMF task model and its properties. Then an example is shown. Finally the applicability of GMF analysis method on DGMF is discussed.

\subsubsection{DGMF Definitions and Properties}

A DGMF task $G_{i}$ is a vector composed of $N_{i}$ frames $F_{i}^{j}$, with $1 \leq j \leq N_{i}$. Each frame is a job of the same task $G_{i}$. Frames have some parameters inherited from GMF:

- $E_{i}^{j}$ is the Worst Case Execution Time (WCET) of $F_{i}^{j}$.

- $D_{i}^{j}$ is the relative deadline of $F_{i}^{j}$.

- $P_{i}^{j}$ is min-separation of $F_{i}^{j}$, defined as the minimum time separating the release of $F_{i}^{j}$ and the release of $F_{i}^{j+1}$.

A DGMF task also has a GMF period [6] inherited from the original task model. For DGMF task $G_{i}$ with $N_{i}$ frames, the GMF period $P_{i}$ of $G_{i}$ is:

$$
P_{i}=\sum_{j=1}^{N_{i}} P_{i}^{j}
$$

Frames also have some parameters and notations specific to the DGMF task model:

- $[U]_{i}^{j}$ is a set of $(R, S, B)$ tuples denoting shared resource critical sections. $F_{i}^{j}$ asks for access to resource $R$ after it has run $S$ time units of its execution time, and then locks the resource during the next $S$ time units of its execution time. 
- $\left[F_{p}^{q}\right]_{i}^{j}$ is a set of predecessor frames, i.e. frames from any other DGMF tasks that must complete execution before $F_{i}^{j}$ can be released. A predecessor frame is denoted by $F_{p}^{q}$. Frame $F_{p}^{q}$ can only be in $\left[F_{p}^{q}\right]_{i}^{j}$ if $G_{i}$ and $G_{p}$ have the same GMF period. When a frame $F_{x}^{y}$ precedes $F_{i}^{j}$, the precedence dependency is denoted $F_{x}^{y} \rightarrow F_{i}^{j}$. We have $\forall F_{p}^{q} \in\left[F_{p}^{q}\right]_{i}^{j}, F_{p}^{q} \rightarrow F_{i}^{j} . F_{p}^{q}$ are not the only frames that precede $F_{i}^{j}$. For example, any frame that precedes a $F_{p}^{q} \in\left[F_{p}^{q}\right]_{i}^{j}$, also precedes $F_{i}^{j}$. For $j>1$, we have $F_{i}^{j-1} \rightarrow F_{i}^{j}$.

- $\operatorname{proc}\left(F_{i}^{j}\right)$ is the processor on which $F_{i}^{j}$ is allocated on. Critical section $(R, S, B)$ can be in $[U]_{i}^{j}$ and $[U]_{x}^{y}$, with $F_{i}^{j} \neq F_{x}^{y}$, only if $\operatorname{proc}\left(F_{i}^{j}\right)=$ $\operatorname{proc}\left(F_{x}^{y}\right)$.

- $\operatorname{prio}\left(F_{i}^{j}\right)$ is the fixed priority of $F_{i}^{j}$ (for FP scheduling).

- $r_{i}^{j}$ is the first release time of $F_{i}^{j}$. For the first frame $F_{i}^{1}$, parameter $r_{i}^{1}$ is arbitrary. For the next frames, we have $r_{i}^{j}=r_{i}^{1}+\sum_{h=1}^{j-1} P_{i}^{h}$.

Frames are released cyclically [6]. Furthermore, the first frame to be released by a DGMF task $G_{i}$ is always the first frame in its vector, denoted by $F_{i}^{1}$. Parameter $r_{i}$ denotes the release time of $G_{i}$ and we have $r_{i}=r_{i}^{1}$.

A DGMF tasks set may have several properties. Let use define the Unique Predecessor property and the Cycle Separation property.

Property 1 (Unique Predecessor). Let $F_{i}^{j}$ be a frame of a task $G_{i}$, in a DGMF tasks set. Let $\left[F_{p}^{q}\right]_{i}^{j}$ be the set of predecessor frames of $F_{i}^{j} . F_{i}^{j-1}$ is the previous frame of $F_{i}^{j}$ in the vector of $G_{i}$, with $j>1$. The set of predecessor frames of $F_{i}^{j}$ is the set $\left[F_{p}^{q}\right]_{i}^{j}$ and $F_{i}^{j-1}$ (with $j>1$ ).

A DGMF tasks set is said to respect the Unique Predecessor property if, for all frames $F_{i}^{j}$, there is at most one frame $F_{x}^{y}$, in a reduced set of predecessors of $F_{i}^{j}$, with a global deadline (i.e. $r_{x}^{y}+D_{x}^{y}$ ) greater than or equal to the release time of $F_{i}^{j}$. The reduced set of predecessors, of $F_{i}^{j}$, are predecessors that do not precede another predecessor of $F_{i}^{j}$.

To formally define the Unique Predecessor property, the set of predecessor frames of $F_{i}^{j}$ is denoted by pred $\left(F_{i}^{j}\right)$ such that:

$$
\operatorname{pred}\left(F_{i}^{j}\right)= \begin{cases}{\left[F_{p}^{q}\right]_{i}^{j} \cup\left\{F_{i}^{j-1}\right\}} & \text { with } j>1 \\ {\left[F_{p}^{q}\right]_{i}^{j}} & \text { otherwise. }\end{cases}
$$

Formally the Unique Predecessor property is then defined as follows:

$$
\exists_{\leq 1} F_{x}^{y} \in \operatorname{pred}\left(F_{i}^{j}\right)^{\prime}, r_{x}^{y}+d_{x}^{y} \geq \max \left(\underset{F_{l}^{h} \in \max d\left(F_{i}^{j}\right)}{\max }\left(r_{l}^{h}+E_{l}^{h}\right), r_{i}^{j}\right)
$$

where $\exists_{\leq 1}$ means "there exists at most one", and the set $\operatorname{pred}\left(F_{i}^{j}\right)^{\prime}$ is defined as:

$$
\operatorname{pred}\left(F_{i}^{j}\right)^{\prime}=\operatorname{pred}\left(F_{i}^{j}\right) \backslash\left\{F_{x}^{y} \in \operatorname{pred}\left(F_{i}^{j}\right) \mid \exists F_{k}^{l} \in \operatorname{pred}\left(F_{i}^{j}\right), F_{x}^{y} \rightarrow F_{k}^{l}\right\}
$$


Table 3: DGMF Task Set

\begin{tabular}{|c|c|c|c|c|c|c|c|}
\hline & $E_{i}^{j}$ & $D_{i}^{j}$ & $P_{i}^{j}$ & {$[U]_{i}^{j}$} & {$\left[F_{p}^{q}\right]_{i}^{j}$} & $\operatorname{prio}\left(F_{i}^{j}\right)$ & $\operatorname{proc}\left(F_{i}^{j}\right)$ \\
\hline \multicolumn{8}{|c|}{$G_{1} ; r_{1}=0$} \\
\hline$F_{1}^{1}$ & 1 & 4 & 1 & & $F_{2}^{1}$ & 1 & cpu1 \\
\hline$F_{1}^{2}$ & 1 & 3 & 1 & & & 1 & cpu2 \\
\hline$F_{1}^{3}$ & 1 & 2 & 6 & & & 1 & cpu1 \\
\hline$F_{1}^{4}$ & 1 & 4 & 4 & & $F_{2}^{2}$ & 1 & cpu1 \\
\hline$F_{1}^{5}$ & 4 & 8 & 8 & $(R, 1,3)$ & $F_{2}^{3}$ & 1 & cpu1 \\
\hline \multicolumn{8}{|c|}{$G_{2} ; r_{2}=0$} \\
\hline$F_{2}^{1}$ & 1 & 4 & 8 & & & 2 & cpu1 \\
\hline$F_{2}^{2}$ & 1 & 4 & 4 & & & 2 & cpu1 \\
\hline$F_{2}^{3}$ & 1 & 4 & 4 & & & 2 & cpu1 \\
\hline$F_{2}^{4}$ & 2 & 4 & 4 & $(R, 0,1)$ & & 2 & cpu1 \\
\hline \multicolumn{8}{|c|}{$G_{3} ; r_{3}=4$} \\
\hline$F_{3}^{1}$ & 1 & 2 & 2 & & $F_{4}^{1}$ & 1 & cpu1 \\
\hline$F_{3}^{2}$ & 1 & 2 & 18 & & $F_{4}^{2}$ & 1 & cpu1 \\
\hline \multicolumn{8}{|c|}{$G_{4} ; r_{4}=4$} \\
\hline$F_{4}^{1}$ & 1 & 2 & 2 & & & 2 & cpu1 \\
\hline$F_{4}^{2}$ & 1 & 2 & 18 & & & 2 & cpu1 \\
\hline
\end{tabular}

Property 2 (Cycle Separation). A DGMF task $G_{i}$ is said to respect the Cycle Separation property if:

$$
D_{i}^{N_{i}} \leq r_{i}+P_{i}
$$

The Unique Predecessor and Cycle Separation properties simplify the analysis method of DGMF so both properties are assumed for a DGMF tasks set. Experiments, presented later in this article, will show that they have no impact on the ability to model a real software radio protocol, developed at Thales, with DGMF.

To illustrate the task model defined in this section, the next section shows an example with some DGMF tasks, the parameters of their frames, and a possible schedule of the tasks set.

\subsubsection{DGMF Example}

Consider the DGMF tasks set in Table 3, modeling tasks constrained by a TDMA frame. Frames of $G_{1}$ and $G_{3}$ have a priority of 1 . Frames of $G_{2}$ and $G_{4}$ have a priority of 2 . All frames are allocated on cpu1 except $F_{1}^{2}$, which is allocated on cpu2. Frames $F_{1}^{5}$ and $F_{2}^{4}$ use a shared resource $R$.

Figure 3 shows an example of a schedule produced by the tasks set, over 20 time units. In the figure, the TDMA frame has $1 S$ slot, $2 B$ slots, and $3 T$ slots. Task $G_{2}$ is released at $S$ and $T$ slots. $G_{2}$ releases $G_{1}$ upon completion. $G_{4}$ is released at $B$ slots. $G_{4}$ releases $G_{3}$ upon completion. Release time parameter $r_{i}$ allows us to specify at which slot a task is released for the first time. For 
example task $G_{4}$ is released at time 4 , which is the start time of the first $B$ slot. Notice that precedence dependencies are respected and $F_{4}^{2}$ is blocked by $F_{1}^{5}$ during 1 time unit, due to a shared resource.

\subsubsection{Applicability of GMF Analysis Methods on DGMF}

Schedulability tests exist for GMF tasks. In this section a test is reminded and its applicability to DGMF is discussed.

In 20] a response time based schedulability test for GMF tasks is proposed. This test assumes that tasks are independent and run on a uniprocessor system with a FP preemptive scheduling policy. Furthermore the authors also assume that the Frame Separation property holds: the relative deadline of a frame is less than or equal to its min-separation. Obviously this test cannot be applied to DGMF tasks for the following reasons:

- DGMF tasks have task dependencies

- DGMF frames may be allocated on different processors.

The next section proposes a scheduling analysis method for DGMF by exploiting the transaction model.

\subsection{DGMF Scheduling Analysis Using Transactions}

In the previous section it was shown that GMF analysis methods cannot be applied to DGMF tasks because of task dependencies and the partitioned multiprocessor nature of the system. Two choices are then available to solve this issue:

- Extend GMF schedulability tests

- Transform to another model where schedulability tests exist

The second approach is chosen in this article: DGMF tasks scheduling analysis will be performed by transforming them to transactions. The transaction model is chosen for the following reasons:

- Task dependencies (precedence and shared resource) can be expressed.

- Partitioned multiprocessor systems are considered.

- A independent GMF to transaction transformation is proposed in 28 for uniprocessor systems.

The transformation faces the issue of the difference in semantic between the two models. Indeed, transactions represent tasks related by collectively performed functionalities and timing attributes [7, not individual jobs of a task. The multiframe task models, on the other hand, express individual job parameters. 
TDMA Frame
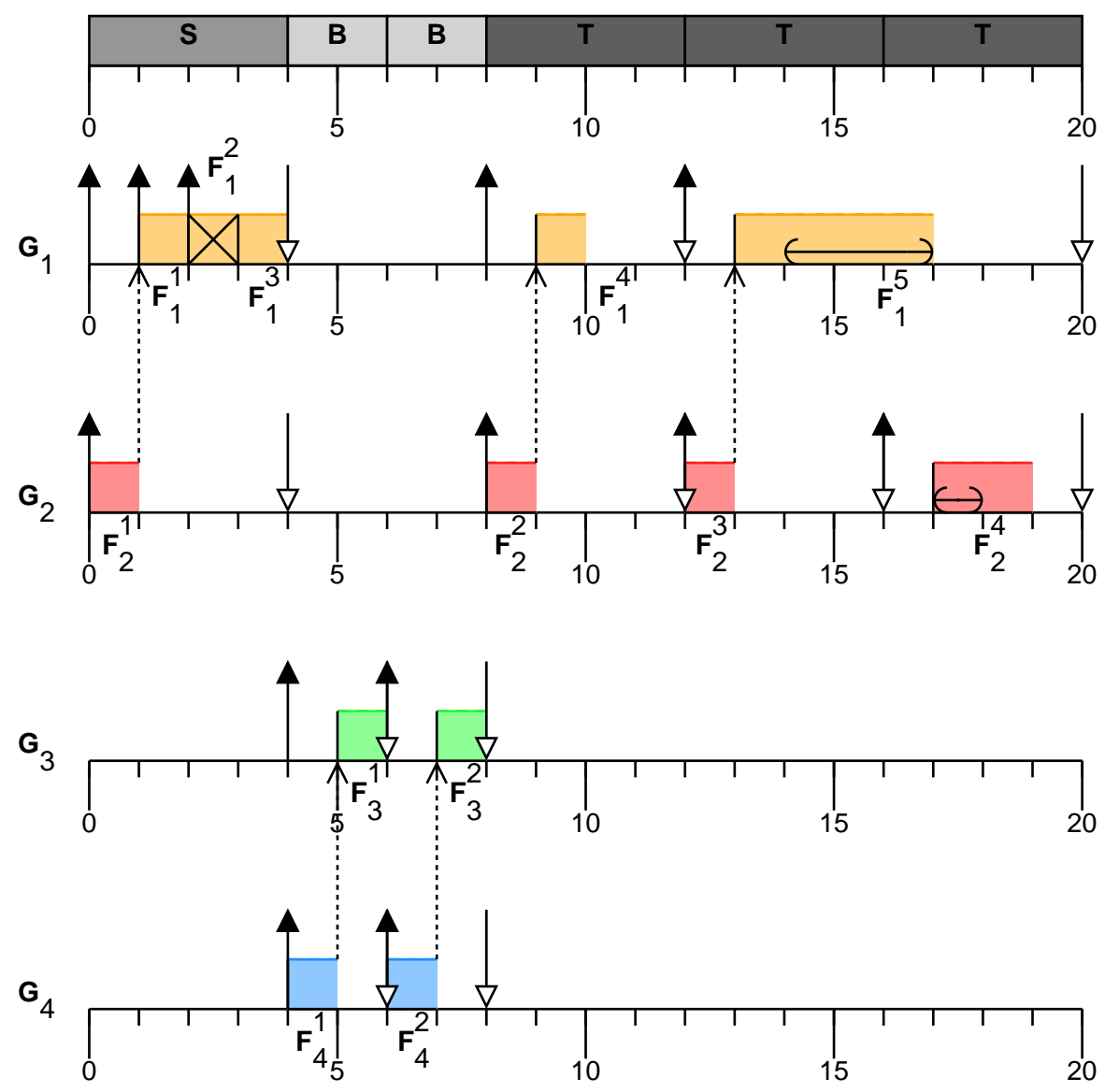

Figure 3: Example of Schedule of DGMF Tasks: Up arrows are frame releases; Down arrows are frame relative deadlines; Dashed arrows are precedence dependencies; Curved arrows are shared resource critical sections where $R$ is used; Crossed frame executes on a different processor 
In the next sections, the transaction model is first reminded. Then the transformation algorithm in [28] is extended for DGMF tasks. Afterwards the DGMF tasks set in Section 4.1.2 is transformed to a transactions set. Finally schedulability tests for transactions are discussed to choose one suitable for transactions resulting from DGMF transformation.

\subsubsection{Transaction Definitions}

According to [29, "a transaction is a group of related tasks (related either through some collectively performed function, or through some shared timing attributes whereby it is convenient to collect these tasks into a single entity)". In [21, transactions are used to model groups of tasks related by precedence dependency. Let us see some definitions and notations for the transaction model, taken from $29,7,21,17,8$.

A transaction is denoted by $\Gamma_{i}$ and its tasks are denoted by $\tau_{i j}$. A transaction is released by a periodic event that occurs every $T_{i}$. A particular instance of a transaction is called a job. A job of a task in a transaction corresponds to a job of the transaction. If the event that releases the $p^{t h}$ job of $\Gamma_{i}$ occurs at $t_{0}$, then the $p^{t h}$ jobs of its tasks are released after or at $t_{0}$. The release time of the first job of $\Gamma_{i}$ is denoted by $r_{i}$. A task $\tau_{i j}$ has the following parameters:

- $C_{i j}$ is the WCET.

- $C_{i j}^{b}$ is the Best Case Execution Time (BCET).

- $O_{i j}$ is the offset, a minimum time that must elapse after the release of the job of $\Gamma_{i}$ before $\tau_{i j}$ is released 21. Otherwise said $\tau_{i j}$ is released at least $O_{i j}$ units of time after $t_{0}$. Value $r_{i j}=r_{i}+O_{i j}$ is the absolute release time of the first job of $\tau_{i j}$.

- $d_{i j}$ is the relative deadline. Value $O_{i j}+d_{i j}$ is the global deadline [17] of $\tau_{i j}$. Value $r_{i}+O_{i j}+d_{i j}$ is the absolute deadline of the first job of $\tau_{i j}$.

- $J_{i j}$ is the maximum jitter, i.e. $\tau_{i j}$ is released in $\left[t_{0}+O_{i j} ; t_{0}+O_{i j}+J_{i j}\right]$.

- $B_{i j}$ is the Worst Case Blocking Time (WCBT) [13] due to shared resources.

- $\operatorname{prio}\left(\tau_{i j}\right)$ is the fixed priority.

- $\operatorname{proc}\left(\tau_{i j}\right)$ is the processor on which $\tau_{i j}$ is allocated on.

- $R_{i j}^{w}$ is the global WCRT, which is the WCRT relative to the release of the transaction [21. A global response time is the response time of a task plus its offset. As a reminder, a response time of a task is relative to its release, in this case its offset.

- $R_{i j}^{b}$ is the global Best Case Response Time (BCRT), which is the BCRT relative to the release of the transaction [21]. 
Tasks may use shared resources in critical sections. A critical section is denoted by $(\tau, R, S, B)$ where $\tau$ is the task using the resource $R$. Task $\tau$ asks for $R$ at $S$ of its execution time, and locks it during the next $B$ units of time of its execution time.

Tasks in a transaction are related by precedence dependencies [17. A precedence dependency between two tasks is denoted by $\tau_{i p} \prec \tau_{i j}$. As a reminder, the precedence dependency means that a job $p$ of $\tau_{i p}$ must complete before a job $p$ of $\tau_{i j}$ can be released. $\tau_{i p}$ (resp. $\tau_{i j}$ ) is called the predecessor (resp. successor) of $\tau_{i j}$ (resp. $\tau_{i p}$ ). According to the precedence dependencies that may exist between tasks, transactions are of different type. This article handles tree-shaped transactions.

Definition 1 (Tree-Shaped Transaction [8]). A tree-shaped transaction $\Gamma_{i}$ has a root task, denoted by $\tau_{i 1}$, which leads to the releases of all other tasks, upon completion. A task $\tau_{i j}$, of a tree-shaped transaction, is said to have at most one immediate predecessor (denoted by pred $\left(\tau_{i j}\right)$ ) that releases it upon completion. A task $\tau_{i j}$ may have several immediate successors (denoted by $\operatorname{succ}\left(\tau_{i j}\right)$ ) that it releases upon completion. The root task $\tau_{i 1}$ has no predecessor.

\subsubsection{DGMF To Transaction}

The DGMF to transaction transformation aims at expressing parameters and dependencies in the DGMF task model as ones in the transaction model. The transformation has three major steps:

- Step 1: Transform independent DGMF to transaction, i.e. consider DGMF tasks as independent and transform them to transactions.

- Step 2: Express shared resource critical sections, i.e. model critical sections in the resulting transaction set and compute WCBTs.

- Step 3: Express precedence dependencies, i.e. model precedence dependencies in the transaction model.

In the following sections, each step is explained in detail.

Independent DGMF to Transaction Step 1 consists in transforming each DGMF task to a transaction by considering DGMF tasks as independent. Algorithm 4.2.2.1 shows the original algorithm proposed in [28] that is extended for DGMF tasks.

The idea behind the algorithm is to transform frames $F_{i}^{j}$ of a DGMF task $G_{i}$ into tasks $\tau_{i j}$ of a transaction $\Gamma_{i}$. Parameters in the transaction model, like WCET $\left(C_{i j}\right)$, relative deadline $\left(d_{i j}\right)$ and priority $\left(\operatorname{prio}\left(\tau_{i j}\right)\right)$, are computed from parameters $E_{i}^{j}, D_{i}^{j}$ and prio $\left(F_{i}^{j}\right)$ from the DGMF model. To transform the min-separation of a frame in the DGMF model, offsets $\left(O_{i j}\right)$ are used in the transaction model. The offset of a task $\tau_{i j}$ is computed by summing the $P_{i}^{h}$ of frames $F_{i}^{h}$ preceding $F_{i}^{j}$ in the vector of $G_{i}$. In the extension of the 
transformation, the release time $r_{i}$ of a DGMF task $G_{i}$ is transformed into the release time $r_{i}$ of a transaction $\Gamma_{i}$.

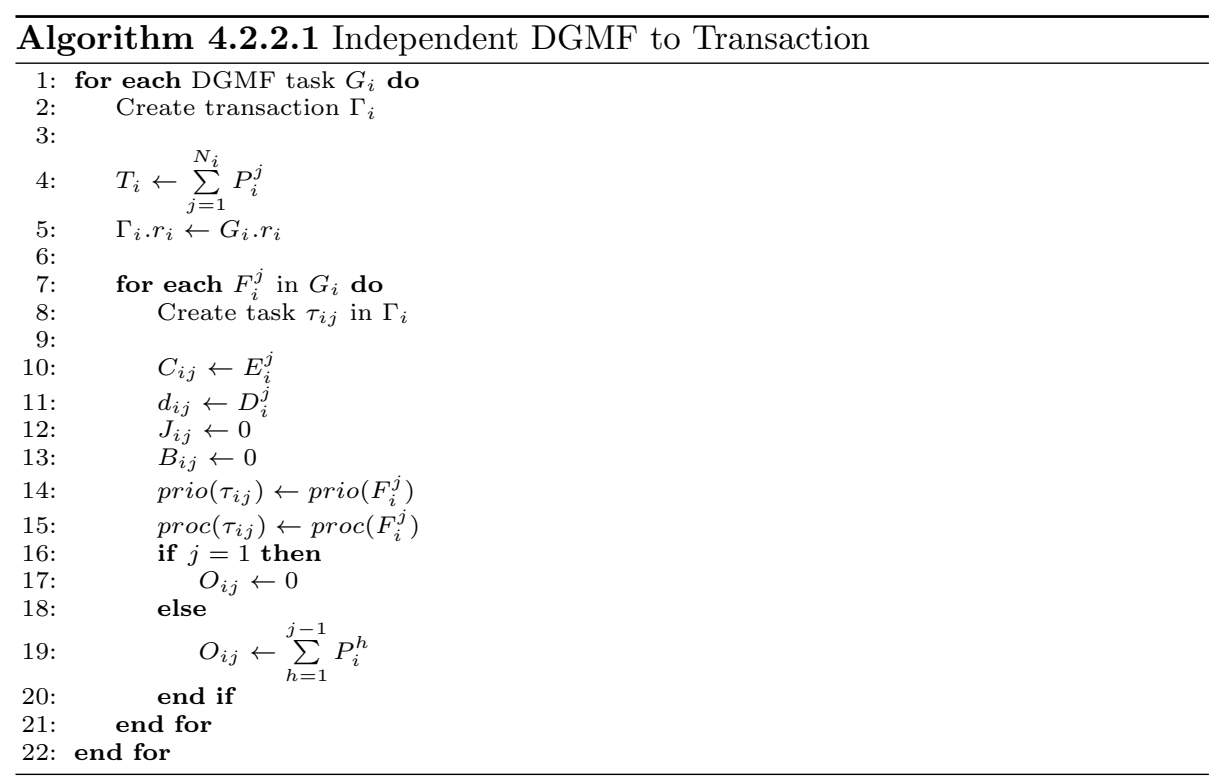

Express Shared Resource Critical Sections In Step 2, the goal is to express critical sections of tasks $\tau_{i j}$ and then to compute their $B_{i j}$. Step 2 is thus divided into 2 sub-steps:

- Step 2.A: Express critical sections

- Step 2.B: Compute worst case blocking times

In Step 2.A, if a critical section is defined for a frame, then the task, corresponding to the frame after transformation, must also have the critical section. If there is a critical section $(R, S, B)$ in $[U]_{i}^{j}$, then a critical section $\left(\tau_{i j}, R, S, B\right)$ must be specified in the transaction set resulting from Step 1.

In Step 2.B, the goal is to compute $B_{i j}$ of a task $\tau_{i j}$, assuming $B_{i j}$ of a task is bounded [13, 12. $B_{i j}$ is computed by considering all shared resource $R$ accessed by $\tau_{i j}$. Then all other tasks $\tau_{x y}$ that may access $R$ are considered too. Since some other tasks $\tau_{x y}$ actually represent jobs of a same DGMF task, not all tasks $\tau_{x y}$ accessing $R$ must be accounted for the computation of $B_{i j}$. Otherwise, it may lead to a pessimistic WCBT.

Consider a task $\tau_{x y}$ that shares a resource $R$ with task $\tau_{i j}$. Let $\left(\tau_{x y}, R, S, B_{\max }\right)$ denote the longest critical section of $\tau_{x y}$. Let $S G\left(\tau_{x y}, \tau_{i j}\right)$ denote the function that returns true if $\tau_{x y}$ and $\tau_{i j}$ result from frames that are part of a same DGMF task. Let $\beta_{i j, R}$ be the set of tasks considered for the computation of $B_{i j}$ for a given $R$. For a given $R$, set $\beta_{i j, R}$ contains a task $\tau_{x y}$ if: 


$$
\begin{aligned}
& \operatorname{proc}\left(\tau_{x y}\right)=\operatorname{proc}\left(\tau_{i j}\right) \wedge \\
& \neg S G\left(\tau_{x y}, \tau_{i j}\right) \wedge \\
& \neg \exists\left(\tau_{k l}, R, S^{\prime}, B^{\prime}\right) \mid\left(S G\left(\tau_{k l}, \tau_{i j}\right) \wedge B^{\prime}>B_{\text {max }}\right)
\end{aligned}
$$

The three conditions have the following meaning:

1. Condition 1 (Equation 6) means that $\tau_{x y}$ must be on the same processor as $\tau_{i j}$.

2. Condition 2 (Equation 7) means that both tasks must not come from frames which are part of a same DGMF task.

3. Finally, for condition 3 (Equation 8) let us suppose that $\tau_{x y}$ results from a frame in a DGMF task $G_{x}$. For a given $R, \tau_{x y}$ is only considered if it has the longest critical section of $R$, among all tasks (with critical sections of $R$ ) that result from frames which are part of $G_{x}$.

Proof of Step 2.B. Frames represent jobs of a same DGMF task. Tasks resulting from frames of a same DGMF cannot block each other since jobs of a same DGMF task cannot block each other. Furthermore, tasks resulting from frames of a same DGMF task, cannot all block another task, as if they are individual tasks, since they represent jobs of a same DGMF task.

Equations for the computation of $B_{i j}$ are then adapted with the new set $\beta_{i j, R}$. The equation in [13] to compute the WCBT with PIP becomes:

$$
B_{i j}=\sum_{R} \max _{\tau_{x y} \in \beta_{i j, R}}\left(\text { Critical section duration of } \tau_{x y}\right)
$$

where $R$ denotes a shared resource.

The equation in 13 to compute the WCBT with PCP becomes:

$$
B_{i j}=\max _{\tau_{x y} \in \beta_{i j, R}, R}\left(D_{x y, R} \mid \operatorname{prio}\left(\tau_{x y}\right)<\operatorname{prio}\left(\tau_{i j}\right), C(R) \leq \operatorname{prio}\left(\tau_{i j}\right)\right)
$$

where $R$ denotes a shared resource, $C(R)$ the ceiling priority [13] of $R$, and $D_{x y, R}$ the duration of the longest critical section of task $\tau_{x y}$ using shared resource $R$.

Express Precedence Dependencies The goal of Step 3 is to model precedence dependencies in the transactions set, with respect to how they are modeled in the transaction model with offsets. Step 3 is divided into three sub-steps:

- Step 3.A: Express precedence dependency, i.e. precedence dependencies between frames are expressed as precedence dependencies between tasks. 
- Step 3.B: Model precedence dependency in the transaction model, i.e. precedence dependencies between tasks are modeled with offsets according to [21]. Precedence dependent tasks must also be in a same transaction.

- Step 3.C: Reduce precedence dependencies, i.e. simplify the transactions set by reducing number of precedence dependencies.

Two kinds of precedence dependency are defined in the DGMF model: intradependency and inter-dependency. A intra-dependency is a precedence dependency that is implicitly expressed between frames of a same DGMF task. Indeed, frames of a DGMF task execute in the order defined by the vector. An interdependency is a precedence dependency between frames belonging to different DGMF tasks.

An intra-dependency in the DGMF set is expressed in the transaction set by a precedence dependency between tasks, representing successive frames, if they are part of a same transaction $\Gamma_{i}$ with $N_{i}$ tasks, resulting from Step 1: $\forall j<N_{i}, \tau_{i j} \prec \tau_{i(j+1)}$. This also ensures that these tasks are part of the same precedence dependency graph, which is important for determining if the transaction is linear, tree-shaped or graph-shaped.

Inter dependencies must also be expressed in the transactions set resulting from Step 1. If a frame $F_{p}^{q}$, corresponding to task $\tau_{p}^{q}$, is in the set of predecessor frames $\left[F_{p}^{q}\right]_{i}^{j}$ of frame $F_{i}^{j}$, corresponding to task $\tau_{i j}$, then a precedence dependency $\tau_{p q} \prec \tau_{i j}$ is expressed.

Proof of Step 3.A. By definition frames of $G_{i}$ are released in the order defined by the vector of $G_{i}$ so $F_{i}^{j}$ precedes $F_{i}^{j+1}\left(j<N_{i}\right)$. Task $\tau_{i j}$ (resp. $\left.\tau_{i(j+1)}\right)$ is the result of the transformation of $F_{i}^{j}$ (resp. $F_{i}^{j+1}$ ), thus by construction we must have $\tau_{i j} \prec \tau_{i(j+1)}$. The same proof is given for $\tau_{p q} \prec \tau_{i j}$, resulting from the transformation of $F_{p}^{q} \in\left[F_{p}^{q}\right]_{i}^{j}$.

In the transaction model, precedence dependencies should be modeled with offsets. This is done in Step 3.B with three algorithms: Task Release Time Modification; Transaction Merge; and Transaction Release Time Modification.

The release time $r_{i j}$ of each task $\tau_{i j}$, in the transactions set, is modified according to precedence dependencies. This enforces that the release time of $\tau_{i j}$ is later or equal to the latest completion time $\left(r_{p q}+C_{p q}\right)$ of a predecessor $\tau_{p q}$ of $\tau_{i j}$. Task release times are changed by modifying offsets because $r_{i j}=r_{i}+O_{i j}$, where $r_{i}$ is the release time of $\Gamma_{i}$. The release time modification algorithm is shown in Algorithm 4.2.2.2. Since the release time of $\tau_{p q}$ may also be modified by this algorithm, release time modifications are made until no more of them occur. Note that when the offset $O_{i j}$ of $\tau_{i j}$ is increased, its relative deadline $\left(d_{i j}\right)$ is shortened and then compared to its WCET $\left(C_{i j}\right)$ to verify if the deadline is missed.

Proof of Algorithm 4.2.2.2. Let us assume $\tau_{p q} \prec \tau_{i j}$. The earliest release time of $\tau_{i j}$ is $r_{i j}$. According to [22], $\tau_{p q} \prec \tau_{i j} \Rightarrow r_{p q}+C_{p q} \leq r_{i j}$ is true. The implication is false if $\neg\left(r_{p q}+C_{p q} \leq r_{i j}\right)$, otherwise said $r_{p q}+C_{p q}>r_{i j}$. Therefore if we 


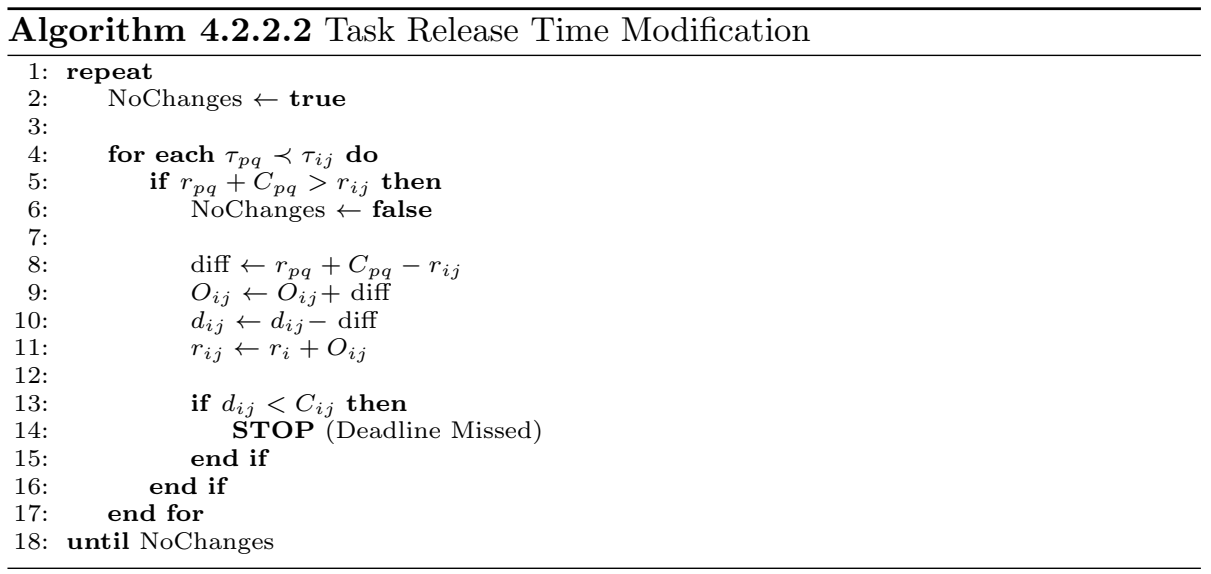

have $\tau_{p q} \prec \tau_{i j}$ then we cannot have $r_{p q}+C_{p q}>r_{i j}$. Thus, for all $\tau_{p q} \prec \tau_{i j}$, release time $r_{i j}$ must be modified to satisfy $r_{p q}+C_{p q} \leq r_{i j}$, if $\tau_{p q} \prec \tau_{i j}$ and $r_{p q}+C_{p q}>r_{i j}$. Since $r_{i j}=r_{i}+O_{i j}$, the offset $O_{i j}$ is increased to increase $r_{i j}$. Relative deadline $d_{i j}$ is relative to $O_{i j}$, thus $d_{i j}$ must be decreased by the amount $O_{i j}$ is increased.

Up until now, the transformation algorithm produces separate transactions even if they contain tasks that have precedence dependencies with other tasks from other transactions. This is not compliant to the modeling of precedence dependencies in [21]. Indeed two tasks with a precedence dependency should be in the same transaction and they should be delayed from the same event that releases the transaction. Two transactions are thus merged into one single transaction if there exists a task in the first transaction that has a precedence dependency with a task in the other transaction:

$$
\exists \tau_{p q}, \tau_{i j} \mid\left(\Gamma_{i} \neq \Gamma_{p}\right) \wedge\left(\tau_{p q} \prec \tau_{i j} \vee \tau_{i j} \prec \tau_{p q}\right)
$$

Merging two transactions consist in obtaining a final single transaction, containing the tasks of both. Algorithm 4.2.2.3 merges transactions two by two until there is no more transaction to merge.

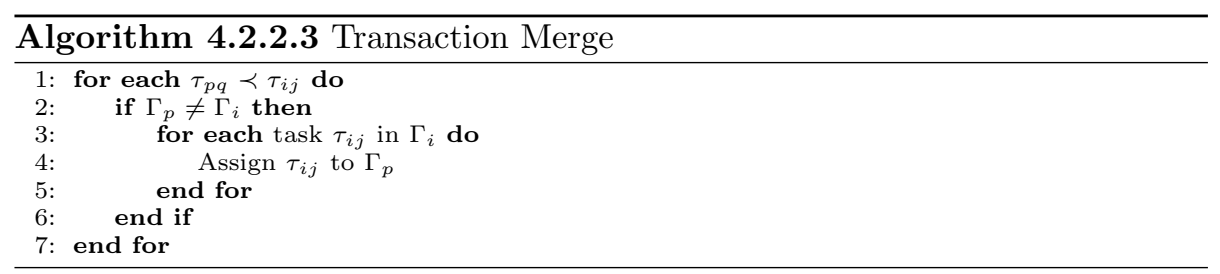

Proof of Algorithm 4.2.2.3. As a reminder, tasks of a transaction are related by precedence dependencies and a task in a transaction is released after the periodic event that releases the transaction. Let us consider two tasks $\tau_{i j}$ and 
$\tau_{p q}$, with $\tau_{p q} \prec \tau_{i j}$. Task $\tau_{i j}$ (resp. $\tau_{p q}$ ) is originally a frame $F_{i}^{j}$ (resp. $F_{p}^{q}$ ). We have $F_{p}^{q} \in\left[F_{p}^{q}\right]_{i}^{j} \Rightarrow P_{i}=P_{p} . G_{i}\left(\right.$ resp. $\left.G_{p}\right)$ is transformed into $\Gamma_{i}\left(\right.$ resp. $\Gamma_{p}$ ) with period $T_{i}\left(\right.$ resp. $\left.T_{p}\right)$. We then have $T_{i}=P_{i}=P_{p}=T_{p}$. Thus $\tau_{i j}$ and $\tau_{p q}$ are released after periodic events of period $T_{i}=T_{p}$. Since $\tau_{p q} \prec \tau_{i j}, \tau_{i j}$ is released after $\tau_{p q}$. Thus $\tau_{i j}$ is released after the periodic event after which $\tau_{p q}$ is released. Therefore $\tau_{i j}$ and $\tau_{p q}$ are released after the same periodic event, that releases transaction $\Gamma_{p}$. Both tasks then belong to $\Gamma_{p}$.

After merging two transactions into $\Gamma_{m}$, the offset $O_{m}^{j}$ of a task $\tau_{m}^{j}$ (originally denoted by $\tau_{o}^{j}$ and belonging to $\Gamma_{o}$ ) is still relative to the release time $r_{o}$ of $\Gamma_{o}$, no matter the precedence dependencies. In $\Gamma_{m}$, each offset must thus be set relatively to $r_{m}$, the release time of $\Gamma_{m}$. Release time $r_{m}$ is computed beforehand. This is done in Algorithm 4.2.2.4.

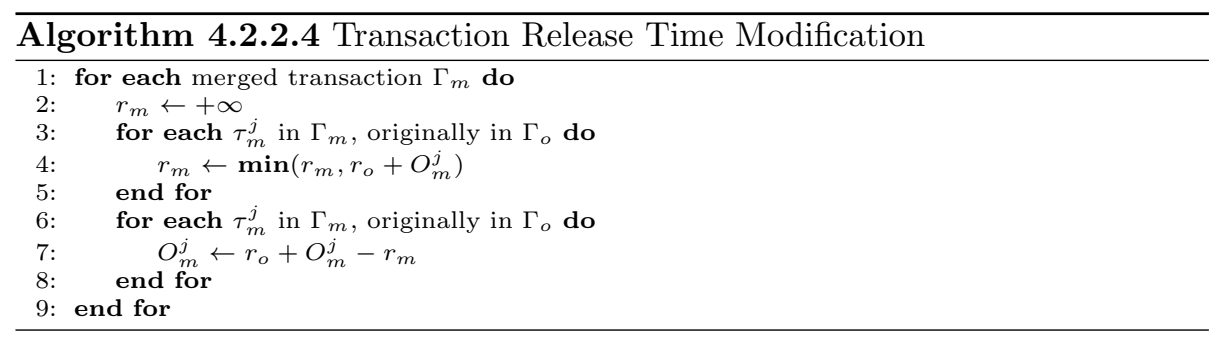

Proof of Algorithm 4.2.2.4. Let $\Gamma_{m}$ be a merged transaction. Tasks in $\Gamma_{m}$ were originally in $\Gamma_{o}$. The event that releases $\Gamma_{m}$ occurs at $r_{m}$, which must be the earliest release time $r_{m}^{j}$ of a task $\tau_{m}^{j}$ in $\Gamma_{m}$, otherwise the definition of a transaction is contradicted. A task $\tau_{m}^{j}$ should be released at $r_{m}^{j}=r_{o}+O_{m}^{j}$. Once $r_{m}$ is computed, when task offsets have not been modified yet, it is possible to have $r_{o}+O_{m}^{j} \neq r_{m}+O_{m}^{j}$. If we assign $r_{m}^{j} \leftarrow r_{m}+O_{m}^{j}$ then $\tau_{m}^{j}$ may not be released at $r_{o}+O_{m}^{j}$. This contradicts the fact that $\tau_{m}^{j}$ should be released at $r_{m}^{j}=r_{o}+O_{m}^{j}$. Therefore $O_{m}^{j}$ must be shortened to be relative to $r_{m}$ : $O_{m}^{j} \leftarrow r_{o}+O_{m}^{j}-r_{m}$. Since $r_{m}=\min _{\tau_{m}^{j} \in \Gamma_{m}}\left(r_{o}+O_{m}^{j}\right)$, the minimum value of $r_{o}+O_{m}^{j}-r_{m}$ is 0 and thus the assignment $O_{m}^{j} \leftarrow r_{o}+O_{m}^{j}-r_{m}$ will never assign a negative value to $O_{m}^{j}$.

Algorithm 4.2.2.4 starts by finding the earliest (minimum) task release time in a merged transaction $\Gamma_{m}$ (as a reminder, $O_{m}^{j}$ is still relative to $r_{o}$ at this moment). The earliest task release time becomes $r_{m}$. The offset $O_{m}^{j}$ of each task $\tau_{m}^{j}$ is then modified to be relative to $r_{m}$. Note that when transactions are merged and all of them have at least one task released at time $t=0$, Algorithm 4.2.2.4 produces the same merged transaction. This is the case for the transaction resulting from the transformation of the DGMF tasks set example (Section 4.1.2), which was used to model tasks constrained by a TDMA frame.

In Step 3.C, the transactions set can be simplified by reducing the number of precedence dependencies expressed in Step 3.A. This could not be done in Step 
3.A, before Step 3.B, because we did not yet know the latest completion time of a task's predecessors. Now that offsets have been modified, some precedence dependencies can be reduced. Reducing precedence dependencies has the effect of reducing the number of immediate predecessors/successors of a task.

Algorithm 4.2.2.5 iterates through tasks with more than 1 predecessor. For a specific task $\tau_{i j}$, the algorithm reduces predecessors $\tau_{i q}$ that have a global deadline (i.e. $O_{i q}+d_{i q}$ ) smaller than the offset $O_{i j}$ of $\tau_{i j}$. This is the first precedence dependency reduction. The algorithm also reduces redundant precedence dependencies, similar to a graph reduction algorithm 30. A precedence dependency is said redundant if it is already expressed by some other precedence dependency. Redundancy is due to the transitivity of precedence dependency. For example, if $\tau_{i q} \prec \tau_{i j}$ and $\tau_{i q}^{\prime} \prec \tau_{i j}$ were expressed previously, and we have $\tau_{i q} \prec \tau_{i q}^{\prime}$ due to some other expressed precedence dependencies and the transitivity of precedence dependency, then $\tau_{i q} \prec \tau_{i j}$ is reduced.

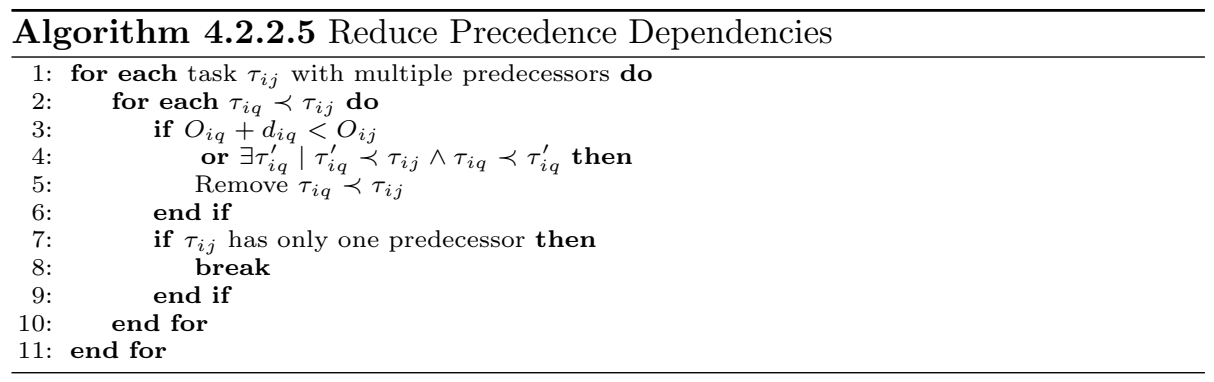

Proof of Algorithm 4.2.2.5. Let us assume $\tau_{i q} \prec \tau_{i j}$ and $O_{i q}+d_{i q}<O_{i j}$. By definition $t_{0}+O_{i j}$ is the earliest release time of a job of $\tau_{i j}$, corresponding to the job of $\Gamma_{i}$ released at $t_{0}$. For the job of $\Gamma_{i}$ released at $t_{0}$, the absolute deadline of a corresponding job of $\tau_{i q}$ is $t_{0}+O_{i q}+d_{i q}$. The job of $\tau_{i q}$ must finish before $t_{0}+O_{i q}+d_{i q}$ and $\tau_{i j}$ is released at earliest after $t_{0}+O_{i q}+d_{i q}$ since $O_{i q}+d_{i q}<O_{i j}$. Thus the precedence dependency constraint $\tau_{i q} \prec \tau_{i j}$ is already encoded in the relative deadline $d_{i q}$ of $\tau_{i q}$. Tasks in a transaction are related by precedence dependency so $\tau_{i j}$ must have at least one predecessor.

The next section shows an example of a DGMF to transaction transformation.

\subsubsection{Transformation Example}

The DGMF tasks set in Section 4.1 .2 is transformed into a transaction $\Gamma_{1}$ of period $T_{1}=20$. Tasks of transaction $\Gamma_{1}$ are defined with parameters shown in Table 4. PCP [13] is assumed for computation of $B_{i j}$. Tasks are allocated on cpu1 except $\tau_{12}$, which is allocated on cpu2. Figure 4 shows the precedence dependency graph of tasks. An example of a schedule over 20 time units is shown in Figure 5. Notice that the schedule of tasks in Figure 5 is the same as the schedule of frames in Figure 3. 
Table 4: Transaction from DGMF Transformation

\begin{tabular}{|l|l|l|l|l|l|l|l|}
\hline & $C_{i j}$ & $O_{i j}$ & $d_{i j}$ & $J_{i j}$ & $B_{i j}$ & $\operatorname{prio}\left(\tau_{i j}\right)$ & $\operatorname{proc}\left(\tau_{i j}\right)$ \\
\hline$\tau_{11}$ & 1 & 1 & 3 & 0 & 0 & 1 & cpu1 \\
\hline$\tau_{12}$ & 1 & 2 & 2 & 0 & 0 & 1 & cpu2 \\
\hline$\tau_{13}$ & 1 & 3 & 1 & 0 & 0 & 1 & cpu1 \\
\hline$\tau_{14}$ & 1 & 9 & 3 & 0 & 0 & 1 & cpu1 \\
\hline$\tau_{15}$ & 4 & 13 & 7 & 0 & 0 & 1 & cpu1 \\
\hline$\tau_{21}$ & 1 & 1 & 4 & 0 & 0 & 2 & cpu1 \\
\hline$\tau_{22}$ & 1 & 8 & 4 & 0 & 0 & 2 & cpu1 \\
\hline$\tau_{23}$ & 1 & 12 & 4 & 0 & 0 & 2 & cpu1 \\
\hline$\tau_{24}$ & 2 & 16 & 4 & 0 & 3 & 2 & cpu1 \\
\hline$\tau_{31}$ & 1 & 5 & 1 & 0 & 0 & 1 & cpu1 \\
\hline$\tau_{32}$ & 1 & 7 & 1 & 0 & 0 & 1 & cpu1 \\
\hline$\tau_{41}$ & 1 & 4 & 2 & 0 & 0 & 2 & cpu1 \\
\hline$\tau_{42}$ & 1 & 6 & 2 & 0 & 0 & 2 & cpu1 \\
\hline Tick & 0 & 0 & $+\infty$ & 0 & 0 & 0 & cpu3 \\
\hline Critical Sections \\
\hline \multicolumn{7}{|l}{$\left(\tau_{13}\right.$ R, 1, 3), $\left.\tau_{24} \mathrm{R}, 0,1\right)$} \\
\hline
\end{tabular}

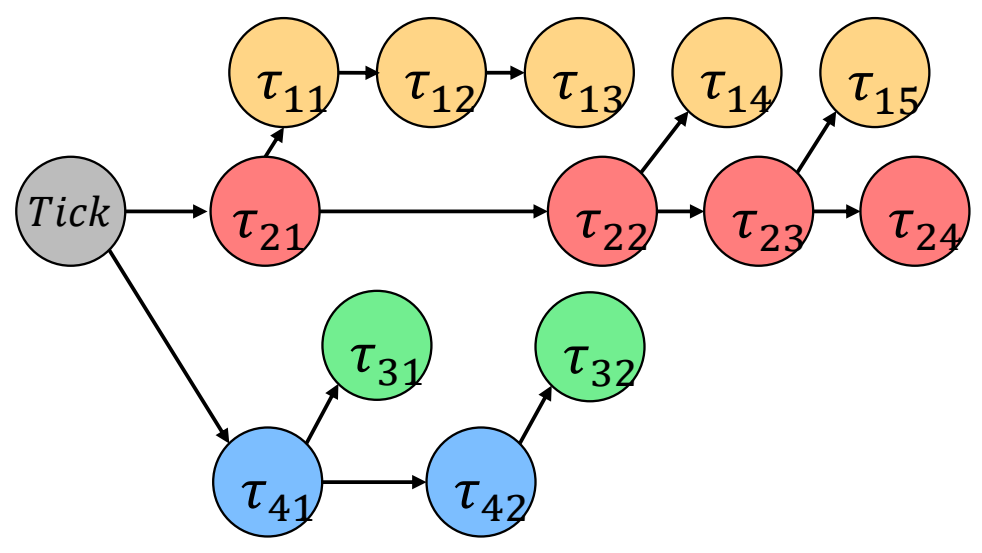

Figure 4: Tasks Precedence Dependency Graph 
TDMA Frame

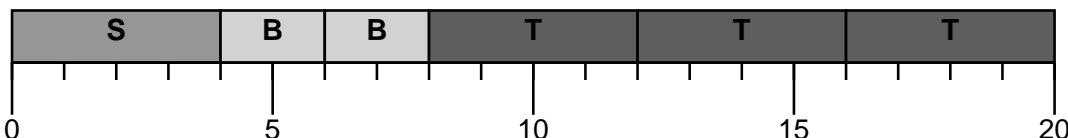

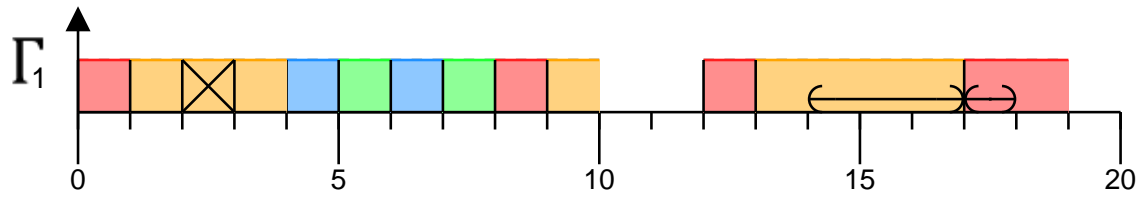

Figure 5: Transaction from DGMF Tasks Transformation: Curved arrows are shared resource critical sections; Tasks execute on same processor except the crossed task

Task Tick represents a ghost root task (defined below) to simplify the analysis, as proposed by [8]. In the specific example, Tick can represent the first TDMA tick that starts the whole TDMA frame. Therefore the ghost root task ensures that all tasks, constrained by the TDMA frame, are part of the same precedence dependency graph. For completeness, parameters of Tick are also given in Table 4. Conform to its definition, Tick is allocated alone on a processor (сри3). It has an execution time of 0 , and offset of 0 , an infinity deadline, no jitter, a blocking time of 0 , and its priority does not matter since it is allocated alone on сриз.

Definition 2 (Ghost Root Task). A ghost task is allocated alone on a processor and is modeled only for the purpose of the analysis. It has a BCET and WCET of 0 , an offset of 0 , an infinity deadline, no jitter, a WCBT of 0 , and its priority does not matter since it is allocated alone on a processor. In a transaction, a ghost root task is a ghost task that precedes all other tasks, and does not have any predecessor.

The next section determines the characteristics of the transaction given by the transformation example presented in this section, and in the general case. A suitable schedulability test is also discussed.

\subsubsection{Assessing Schedulability of Resulting Transactions}

The task parameters in Table 4 , the precedence dependency graph in Figure 4 and the schedule in Figure 5 show that the transaction presented in the last section has the following characteristics:

- Tree-shaped

- Tasks may be non-immediate as defined below. 
Definition 3 (Non-Immediateness). A task $\tau_{i x}$ and its immediate successor task $\tau_{i y}$ are said to be non-immediate tasks if $\tau_{i x}=\operatorname{pred}\left(\tau_{i y}\right) \wedge O_{i y}>O_{i x}+C_{i x}^{b}$. Task $\tau_{i x}$ is called a non-immediate predecessor and $\tau_{i y}$ a non-immediate successor.

A non-immediate task is thus one that is not necessarily immediately released by its predecessor. It is released at an earliest time $t$ if the predecessor completes before or at $t$, but immediately by the predecessor if the predecessor completes after $t$.

In the general case, tree-shaped transactions with non-immediate tasks are the results of the DGMF to transaction transformation, if the DGMF tasks set has the Unique Predecessor property:

Theorem 1. A DGMF tasks set with the Unique Predecessor property (Property 1) is transformed into a transaction set without tasks that have more than one predecessor.

Proof. Let a DGMF tasks set have the Unique Predecessor property. A frame $F_{i}^{j}$ with inter and intra-dependencies is transformed into a task $\tau_{i j}$ with several immediate predecessors. Task $\tau_{i j}$ has several immediate predecessors that correspond to predecessor frames of $F_{i}^{j}$. Let $\tau_{i y}$ be an immediate predecessor of $\tau_{i j}$.

Algorithm 4.2.2.5 removes the precedence dependency $\tau_{i y} \prec \tau_{i j}$, if it is redundant, i.e. if $\tau_{i y}$ does not result from a frame in the reduced set of predecessors (see Property 1) of $F_{i}^{j}$. From now, let us consider that redundant precedence dependencies have been removed. Otherwise said, only frames of the reduced set of predecessors of $F_{i}^{j}$ are considered, as well as tasks that result from them.

At most one predecessor frame $F_{x}^{y}$ of $F_{i}^{j}$ can have a global deadline (i.e. $\left.r_{x}^{y}+D_{x}^{y}\right)$ greater than the release time $r_{i}^{j}$ of $F_{i}^{j}$. By construction, at most one immediate predecessor $\tau_{i y}$ of $\tau_{i j}$ (resulting from $F_{x}^{y}$ and assigned to the same transaction as $\tau_{i j}$ ) can have a global deadline greater than the offset of $\tau_{i j}$ (i.e. $O_{i y}+d_{i y} \geq O_{i j}$ ). Algorithm 4.2.2.5 removes a precedence dependency $\tau_{i y} \prec \tau_{i j}$ if $O_{i j}>O_{i y}+d_{i y}$. Since there is at most one immediate predecessor $\tau_{i y}$ of $\tau_{i j}$ that has $O_{i y}+d_{i y} \geq O_{i j}$, all other immediate predecessors will be reduced until task $\tau_{i j}$ has at most one immediate predecessor.

This proves that tree-shaped transactions result from transformation of DGMF tasks respecting the Unique Predecessor property. The transformation example in Section 4.2.3 showed that there is at least one case where there are nonimmediate tasks in the transactions set resulting from the transformation.

To assess schedulability of transactions that are the result of the transformation, a schedulability test applicable to tree-shaped transactions with nonimmediate tasks must be applied. This section (Section (4) does not focus on proposing a schedulability test for tree-shaped transactions with non-immediate tasks. The test will be the subject of Section 5

Furthermore, without knowledge that the tasks represent frames initially, the schedulability test considers that it is possible for job $k$ of a task $\tau_{i 1}$ representing the first frame of a DGMF task $G_{i}$, to interfere with job $k-1$ of a task $\tau_{i N_{i}}$ 
representing the last frame of the same DGMF task $G_{i}$. In practice, this is not possible because job $k$ of $\tau_{i 1}$ executes after job $\tau_{i N_{i}}$ because frames represent jobs of a DGMF task. If the Cycle Separation property is met, then job $k$ of $\tau_{i 1}$ will only interfere job $k-1$ of $\tau_{i N_{i}}$ if $\tau_{i N_{i}}$ misses its deadline. This is why the Cycle Separation property is assumed.

The following section shows some experiments that evaluate the DGMF modeling and the transformation of DGMF tasks to transactions.

\subsection{Experiment and Evaluation}

The DGMF scheduling analysis method is implemented in the Cheddar scheduling analysis tool. Therefore the DGMF and GMF task models, transaction model, transformation of DGMF tasks to transactions, and some schedulability tests for transactions are implemented in this tool 1 .

In this section, three aspects of the DGMF to transaction transformation are evaluated through experiments. The following sections first evaluates the transformation correctness, then the transformation time performance, and finally the DGMF modeling and transformation scalability, when it is applied to a real case-study.

\subsubsection{Transformation Correctness}

The transformation correctness is evaluated by scheduling simulation performed on randomly generated system architecture models. Cheddar provides a module that is able to generate random architecture models. The generator is updated so DGMF tasks can be produced.

The generator respects some parameters defined by the user. The user may define the number of entities in the model, i.e. processors, DGMF tasks, frames, shared resources, critical sections, and precedence dependencies. The user may also define the scheduling policy, the number of DGMF tasks with the same GMF period (called "synced DGMF tasks"), and the GMF period for such tasks. The generator avoids inconsistencies in the model (e.g. DGMF tasks with no frames). It also produces precedence dependencies in a way that avoids deadlocks during the scheduling simulation.

By using an architecture generator of Cheddar, DGMF task sets are randomly generated. The varying generator parameters are: 2 to 5 DGMF tasks, as many frames as tasks and up to 10 for each number of tasks, 1 to 3 shared resources, as many critical sections as frames, as many precedence dependencies as frames, a GMF Period between 10 to 50, and 50\% of DGMF tasks with the same GMF Period. From these parameters, 540 DGMF architecture models are generated. Each of them is transformed to an architecture model with transactions.

\footnotetext{
${ }^{1}$ All sources available at http://beru.univ-brest.fr/svn/CHEDDAR/trunk/src/ Examples of use available at http://beru.univ-brest.fr/svn/CHEDDAR/trunk/project_examples/ dgmf_sim/ and http://beru.univ-brest.fr/svn/CHEDDAR/trunk/project_examples/ wcdops+_nimp/
} 
The number of tasks, critical sections, and precedence dependencies in the transaction models are equal to the number of frames, critical sections, and precedence dependencies in the DGMF models. The number of output transactions is less than the number of input DGMF tasks, since some transactions are merged into a same one, during the transformation.

Both DGMF and transaction models are then simulated in the feasibility interval proposed in [31]. Schedules are then compared. Each DGMF schedule is strictly similar to the corresponding transaction schedule. Along with the proofs in Section 4.2.2 this experiment enforces the transformation correctness and its implementation correctness in Cheddar.

\subsubsection{Transformation Time Performance}

In the Cheddar implementation, the time complexity of the transformation algorithm depends on two parameters: $n_{F}$ the number of frames, and $n_{D}$ the number of task dependencies (both precedence and shared resource). The complexity of the transformation is $O\left(n_{D}^{2}+n_{F}\right)$. When $n_{F}$ is the varying parameter, the complexity of the algorithm should be $O\left(n_{F}\right)$. When $n_{D}$ is the varying parameter, the complexity of the algorithm should be $O\left(n_{D}^{2}\right)$ due to Algorithm 4.2 .2 .2 which has the same complexity as the algorithm in [22]. The experiment in this section checks that the duration of the transformation, implemented in Cheddar, is consistent with these time complexities. Measurements presented below are taken on a Intel Core i5 @ $2.40 \mathrm{GHz}$ processor.

Figure 6 shows the transformation duration by the number of frames. The number of precedence dependencies is set to 0 (i.e. no intra-dependencies either). Figure 6 shows that the duration is polynomial when the number of frames varies. One can think that this result is inconsistent with the time complexity of the algorithm, which should be $O\left(n_{F}\right)$ when $n_{F}$ is the varying parameter. In practice, the implementation in Cheddar introduces a loop to verify that a task is not already present in the system's tasks set. Thus the time complexity of the implementation is $O\left(n_{F}^{2}\right)$.

Figure 7 shows the transformation duration by the number of precedence dependencies. The number of frames is set to 1000, the number of DGMF tasks to 100 , and the number of shared resources to 0 . In the Cheddar implementation it does not matter which dependency parameter (precedence or shared resource) varies to verify the impact of $n_{D}$. Indeed, all dependencies are iterated through once and precedence dependency has more impact on the transformation duration. Since there are 1000 frames and 100 DGMF tasks, the minimum number of precedence dependencies starts at 900, due to intra-dependencies.

The transformation duration should be polynomial when the number of precedence dependencies vary but this can not be noticed in Figure 7 due to the scale of the figure. Indeed, there is already a high minimum number of precedence dependencies starting at 900 . The local minimum of the polynomial curve is at 0, like in Figure 6. The curve is a polynomial curve and the result is consistent with the complexity, which is $O\left(n_{D}^{2}\right)$ when $n_{D}$ is the varying parameter. 


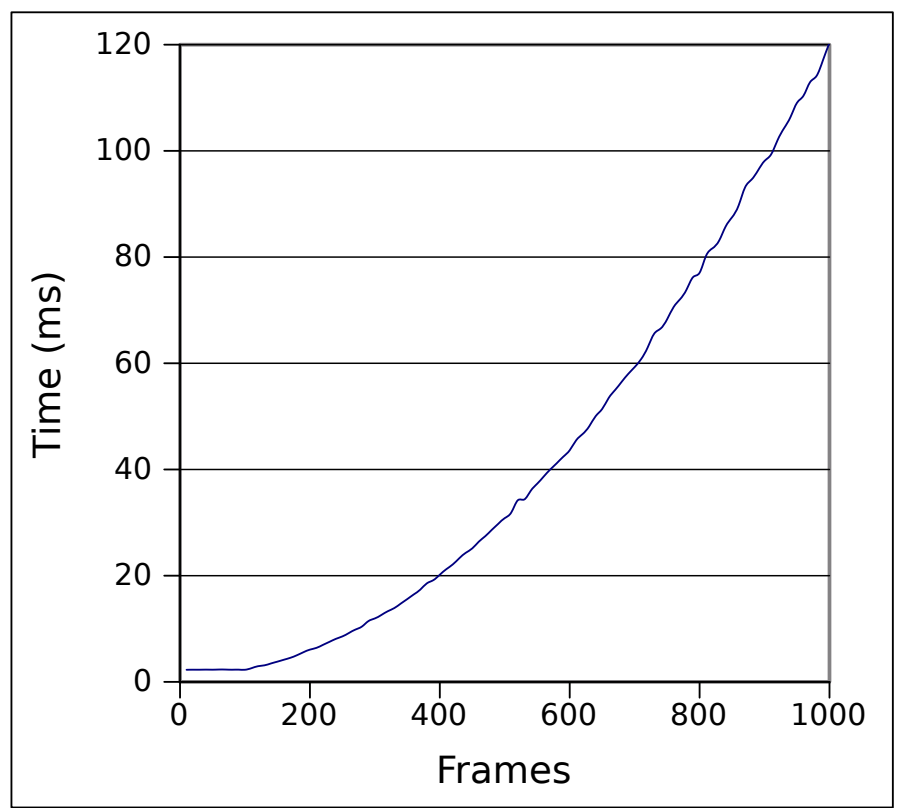

Figure 6: Transformation Duration by Number of Frames

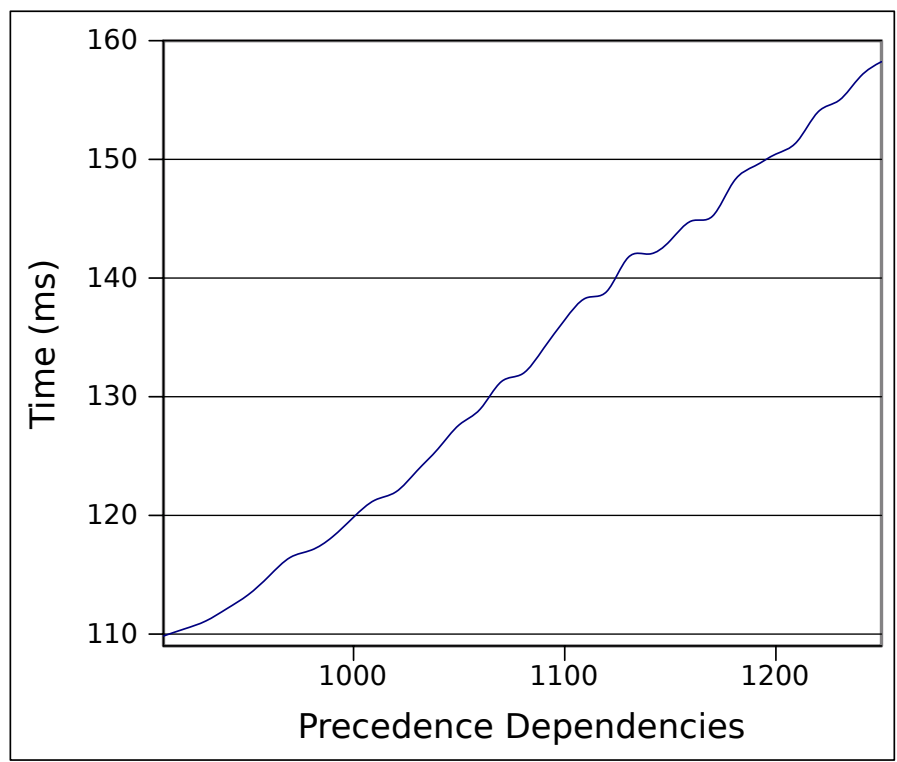

Figure 7: Transformation Duration by Number of Precedence Dependencies 
Overall a system with no dependency, 1000 frames, and 100 DGMF tasks, takes about $120 \mathrm{~ms}$ to be transformed on the computer used for the experiment. A system with 1100 precedence dependencies, 1000 frames, and 100 DGMF tasks, takes less than $160 \mathrm{~ms}$ to be transformed. The transformation duration is acceptable for Thales. Indeed, a typical Thales system has 10 tasks and a TDMA frame of 14 slots $(1 \mathrm{~S}, 5 \mathrm{~B}, 8 \mathrm{~T})$. There would be a maximum of 140 frames $(14 \times 10)$, and 256 precedence dependencies $(10 \times 14-10+9 \times 14)$ if all tasks are part of a same precedence dependency graph, where a task releases at most one task, and a first task is released at each slot.

\subsubsection{Case-Study Modeling with DGMF}

The scalability of the DGMF task model, and its transformation to transaction, is assessed by applying DGMF for the modeling of a real software radio protocol developed at Thales. The case-study is implemented with 8 POSIX threads [32] on a processor called GPP1, and 4 threads on a processor called GPP2. The threads are scheduled by the SCHED_FIFO scheduler of Linux (preemptive FP policy). The threads have precedence dependencies, whether they are on the same processor or not. For example threads on GPP1 may make blocking calls to functions handled by threads on GPP2. When a thread makes a blocking call to a function, it has to wait for the return of the function, before continuing execution. There is one thread on GPP2 dedicated to each thread on GPP1 that may make a blocking call.

The TDMA frame of the case-study has $1 S$ slot, $5 B$ slots, and $8 T$ slots. The threads are released at different slots. The release logic is a thread dedicated to reception is released at a $R x$ slot, while a thread dedicated to transmission is released so its deadline coincides with the start of a $T x$ slot.

In total, there are 36 jobs from 8 threads on GPP1, and 4 threads on GPP2. The threads, their precedence dependencies, and the time parameters of their jobs are illustrated in Figure 8 .

The case-study is modeled with a DGMF task model of 43 frames. There are more frames than the 36 jobs because some jobs that make a blocking call to a function, are divided into several frames. After the transformation, a transaction of 44 tasks is the result. The extra task, compared to the 43 frames, is a ghost root task added by a test like [8]. The transaction is illustrated in Figure 9 .

Notice that different jobs of a thread, released at different slots, become non-immediate tasks in the transaction, related by precedence dependency (e.g. $R S_{-} B 1 \prec R S_{-}$B2). Furthermore, a thread that makes a blocking call, to a function handled by a thread on GPP2, is divided into several frames, and then several tasks (e.g. Frame_Cycle becomes $F C_{-} S 1_{-} 1 \prec F C_{-} S 1_{-} 2 \prec F C_{-} S 1_{-}$3) .

Furthermore, it may seem that some tasks should have two predecessors. For example we should have $A B_{-} B 1_{-} 1 \prec A B_{-} B \mathscr{2}_{-} 1$ because these two tasks represent two jobs of a same thread. We should also have $R x_{-} S l o t \_B 2 \prec A B \_B \mathscr{Z}_{-} 1$ since these two tasks represent jobs of two threads with a precedence dependency. But since the offset of $A B \_B \mathscr{2}_{-} 1$ is strictly greater than the deadline of $R x_{-} S l_{\text {ot_B }} B$, 


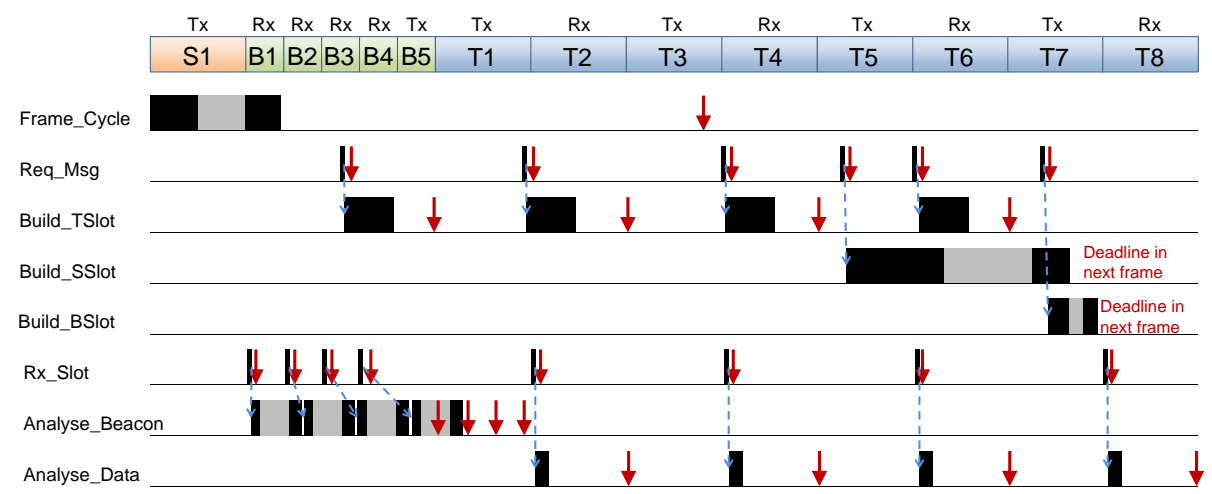

Figure 8: TDMA Frame and Threads of Real Case-Study: Line $=$ Instances of a thread; Down arrow $=$ Deadline; Dashed arrow $=$ Precedence; Black $=$ Exec on GPP1; Gray = Exec on GPP2; 36 jobs in total from 8 threads on GPP1 and 4 threads on GPP2; Sizes not proportional to time values

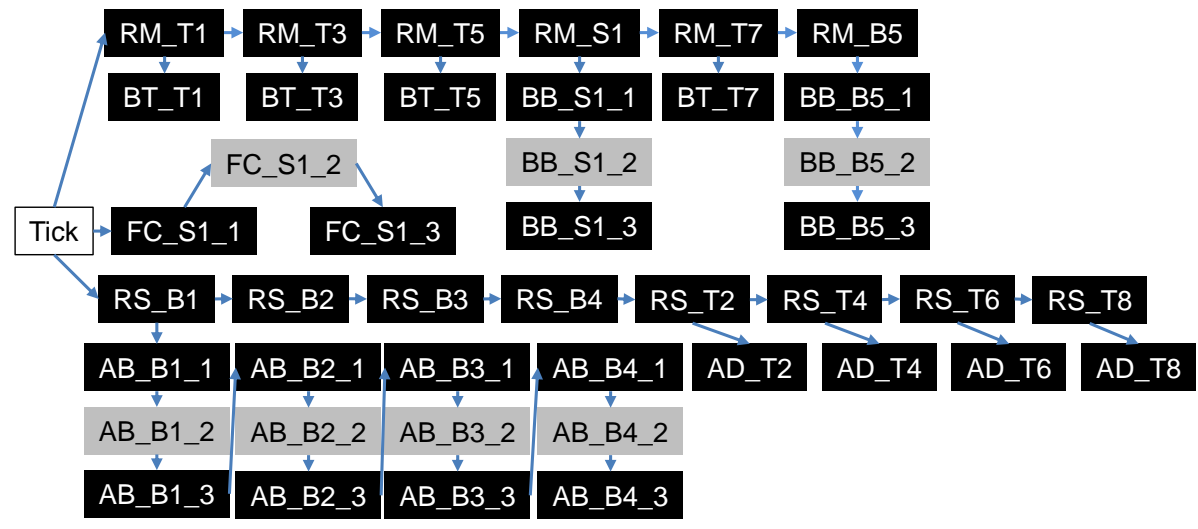

Figure 9: Tree-Shaped Transaction of a Real Case-Study: Black tasks on GPP1, gray tasks on GPP2, Tick task is ghost root task; Task nomenclature is [Name]_[Slot]_[Part (Optional)]; Abbreviated names are RM $=$ Request_Msg, $\mathrm{BT}=$ Build_TSlot, $\mathrm{BB}=$ Build_BSlot, $\mathrm{BS}=$ Build_SSlot, $\mathrm{FC}=$ Frame_Cycle, $\mathrm{RS}=R x_{-}$Slot, $\mathrm{AB}=$ Analyse_Beacon, $\mathrm{AD}=$ Analyse_Data 
the precedence dependency $R S_{-} B 2 \prec A B_{-} B 2_{-} 1$ is reduced by the DGMF transformation. Other cases of tasks with multiple predecessors are due to the same kind of precedence dependencies as the example. Multiple predecessors are thus reduced to one in the same way as the example, by the transformation. In the end the resulting transaction is tree-shaped.

In general, the more jobs of a thread there are, the more frames there are. Similarly, the more there are blocking calls to functions allocated on other processors, the more frames there are.

In the next section, we propose a schedulability test for tree-shaped transactions with non-immediate tasks. Such type of transactions can be the result of DGMF transformation.

\section{Schedulability Analysis of Tree-Shaped Trans- actions With Non-Immediate Tasks}

Schedulability tests for tree-shaped transactions have been proposed by [8] but they do not handle non-immediate tasks. Non-immediate tasks need specific schedulability analysis. Therefore, in this section, a schedulability test is proposed for tree-shaped transactions with non-immediate tasks. The test is called WCDOPS+NIM and it extends the WCDOPS+ test proposed in [8]. The proposed test completes the general DGMF analysis method presented in the previous section.

In the following sections, first the consequence of non-immediateness is shown. Then the WCDOPS+NIM test is exposed. First, experiments show some simulations that compare the original WCDOPS+ test to our test. Finally, our test is applied to a real case-study from Thales.

\subsection{Applicability of WCDOPS+ on Non-immediate Tasks}

Based on a software radio protocol architecture, we have shown in [33, 34, that if WCDOPS+ is applied directly to non-immediate tasks, then interference is underestimated. Indeed, the test does not consider that there are tasks that are not released immediately, therefore it omits some combinations of tasks that may interfere together some other task. This means WCRT computation is optimistic.

We proposed a possible solution to this underestimation problem in 33 , 34. We proposed to model non-immediateness between two tasks by ghost intermediate tasks. Before defining a ghost intermediate task, the terminology

of tree-shaped transaction entities must first be updated due to the existence of non-immediate tasks.

Definition 4 (Direct Predecessor and Successor in Tree-shaped Transaction). A task $\tau_{i j}$ is said to have one direct predecessor, denoted by $\operatorname{pred}\left(\tau_{i j}\right)$, and a set of direct successors, denoted by $\operatorname{succ}\left(\tau_{i j}\right)$. A task $\tau_{i x}$ is $\operatorname{pred}\left(\tau_{i j}\right)$ (resp. in 
$\left.\operatorname{succ}\left(\tau_{i j}\right)\right)$ if there is no task $\tau_{i y}$ such that $\tau_{i x} \prec \tau_{i y} \prec \tau_{i j}$ (resp. $\left.\tau_{i j} \prec \tau_{i y} \prec \tau_{i x}\right)$. For the root task of a tree-shaped transaction, $\operatorname{pred}\left(\tau_{i 1}\right)$ is undefined.

The concept of ghost tasks is introduced in 8] (Definition 2). The concept of intermediate tasks is proposed in 35. An intermediate task represents some extra execution time or message transmission time. A ghost intermediate task can be defined as follows:

Definition 5 (Ghost Intermediate Task). A ghost intermediate task $\tau_{\text {ixy }}$ is a task between $\tau_{i x}$ and its non-immediate direct successor $\tau_{i y}\left(\tau_{i x} \prec \tau_{i y}\right)$. Precedence dependency $\tau_{i x} \prec \tau_{i y}$ is replaced by $\tau_{i x} \prec \tau_{i x y} \prec \tau_{i y}$. Ghost intermediate task $\tau_{i x y}$ is allocated alone on a processor, modeled only for the ghost intermediate task. Parameters of task $\tau_{i x y}$ are formally defined as follows:

$$
\begin{aligned}
& C_{i x y}=C_{i x y}^{b}=O_{i y}-\left(O_{i x}+C_{i x}^{b}\right) \\
& O_{i x y}=O_{i x}+C_{i x}^{b} \\
& J_{i x y}=R_{i x}^{w}-O_{i x y} \\
& D_{i x y}=\infty \\
& B_{i x y}=0 \\
& \operatorname{prio}\left(\tau_{i x y}\right)=1 \\
& \forall \tau_{k l}, \operatorname{proc}\left(\tau_{i x y}\right) \neq \operatorname{proc}\left(\tau_{k l}\right)
\end{aligned}
$$

Unfortunately if we model non-immediateness with ghost intermediate tasks, WCRT computation becomes pessimistic [33, 34. Consider a task $\tau_{i x}$ and its non-immediate direct successor $\tau_{i y}$. Even if the response time of $\tau_{i x}$ increases, it does not necessarily finish after the earliest release time of $\tau_{i y}$. Task $\tau_{i x}$ then has no impact on the response time of $\tau_{i y}$. With a ghost intermediate task $\tau_{i x y}$ between the two, due to the precedence dependencies, any increase to the response time of $\tau_{i x}$ will increase the response time of $\tau_{i x y}$ and thus $\tau_{i y}$ [33, 34. Therefore the WCRT of non-immediate tasks may be overestimated if we model ghost intermediate tasks.

In the case where $\tau_{i y}$ is released immediately by $\tau_{i x}$ (i.e. case where $\tau_{i x}$ finishes after the earliest release of $\tau_{i y}$ ), then $\tau_{i y}$ executes instead of lower priority tasks. Any higher priority tasks released by the lower priority tasks will not interfere $\tau_{i y}$. Consider now that $\tau_{i y}$ is preceded by a ghost intermediate task $\tau_{i x y}$. While $\tau_{i x y}$ is executing, the lower priority tasks can execute and release the higher priority tasks before $\tau_{i x y}$ finishes. These higher priority tasks may then interfere $\tau_{i y}$. The response time of $\tau_{i y}$ is then impacted by both $\tau_{i x}$ and the interferences, although it is incorrect as shown in [33, 34]. This result shows again how the WCRT of a non-immediate task may be overestimated if we model ghost intermediate tasks.

In conclusion, two problems are observed when applying WCDOPS+ to transactions with non-immediate tasks. First, if applied directly, tasks interference may be underestimated, and thus WCRT computation is optimistic. Second, by modeling non-immediate tasks with ghost intermediate tasks, response 


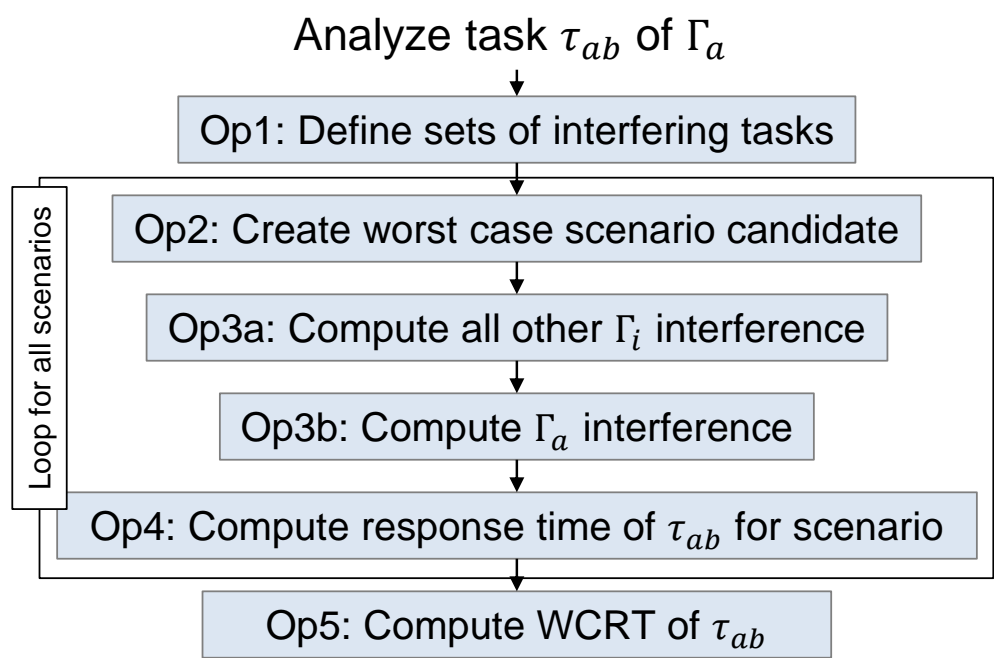

Figure 10: WCDOPS+NIM Overview: Circles indicate key operations

time and tasks interference are overestimated. WCRT computation is then pessimistic. In the following section our test proposes to solve these problems by considering the effects of non-immediateness directly.

\subsection{A Schedulability Test for Non-Immediate Tasks}

To consider the effects of non-immediateness directly, we propose the Worst Case Dynamic Offset with Priority Schemes Plus for Non-Immediate tasks (WCDOPS+NIM) schedulability test, which extends the original WCDOPS+ test. The general approach of the WCDOPS+NIM algorithm is the same as the WCDOPS+ algorithm. The algorithm is illustrated in Figure 10 The system to analyze has some transactions $\Gamma_{i}$. It is assumed that the WCRT of $\tau_{a b}$, belonging to $\Gamma_{a}$, is computed.

Due to the complexity of the mathematical equations and space limitations, we will now only summarize the crucial steps of algorithm. A fully detailed explanation the WCDOPS+NIM test, and its differences with the original test, can be found in [33, 34]. We also provided theorems and proofs to justify the changes we made.

Op1: Define sets of interfering tasks The initial step is to define some task sets to help the analysis of $\tau_{a b}$. For example some tasks of higher priority than $\tau_{a b}$ should be considered to interfere $\tau_{a b}$ as one single task if they are related by precedence dependencies $[8]$.

In order to identify the correct set of tasks, and thus not underestimate the interferences on $\tau_{a b}$, we can consider that non-immediate tasks are preceded by ghost intermediate tasks. We model these ghost intermediate tasks only to 
compute the correct tasks sets in this step. They are not considered in the next steps.

Op2: Create worst case scenario candidate In this step, we create a worst case scenario candidate to compute the response time of of $\tau_{a b}$ within the scenario. The worst case scenario is when some higher priority task $\tau_{a c}$ (also in $\Gamma_{a}$ ) starts the busy period of $\tau_{a b}$. As a reminder, The busy period of a task is the time interval during which the processor executes jobs of the task and jobs of other tasks of higher priority or equal priority [36]. To get the worst scenario, we then start a busy period with all tasks $\tau_{a c}$, hence a worst case scenario candidate. Task $\tau_{a c}$ is chosen by considering tasks that interfere as one single task, computed in the previous step.

When there is a non-immediate higher priority task $\tau_{a c}$, then we consider that it can start the busy period. We also check if the non-immediate task $\tau_{a c}$ can experience jitter, due to its predecessor finishing after the earliest release time of $\tau_{a c}$. Indeed, a non-immediate task should not experience jitter if it starts the busy period, and its predecessor task is of higher priority than the analyzed $\tau_{a b}$ task [33, 34].

Op3a, Op3b: Compute $\Gamma_{i}$ and $\Gamma_{a}$ interference In this step, we compute the interferences from of transactions $\Gamma_{i}$ to $\tau_{a b}$, including $\Gamma_{a}$. When interferences are computed, execution conflicts are considered. Not all sets of tasks defined in Op1 can interfere together. They are then in execution conflict. This is due to precedence dependencies, task priorities, and processor allocations [8].

When there are non-immediate tasks, we check which non-immediate task must be released immediately in the scenario. This will impact the detection of execution conflicts. Indeed, a non-immediate task released immediately by a precedent task should be in the set of the precedent task. Since execution conflicts are detected correctly, the the interferences are not overestimated [33, 34 .

From an implementation point of view, the algorithm computes interferences by exploring the tree-shaped transaction with a depth-first search. Modifications are needed in the exploration algorithm, due to non-immediate tasks [33, 34.

Op4: Compute response time of $\tau_{a b}$ for scenario In this step, the response time of $\tau_{a b}$ is computed for the worst case scenario candidate. We take the longest response time of one of its jobs, since several jobs of $\tau_{a b}$ can be released in the busy period of the scenario. A response time is computed by considering the execution time of $\tau_{a b}$, the interferences it experiences, the jitter it experiences (especially due to preceding tasks), and the WCBT it experiences due to shared resources [8].

If the longest response time comes from the scenario where $\tau_{a c}=\tau_{a b}$ starts the busy period, then $\tau_{a b}$ could have not experienced jitter, thanks to step Op2. Therefore the response time of $\tau_{a b}$ is not overestimated [33, 34]. 
Op5: Compute WCRT of $\tau_{a b}$ In this step, the WCRT of $\tau_{a b}$ is computed by taking the longest response time computed from one of the worst case scenario candidates.

The WCDOPS+NIM test completes the scheduling analysis method for DGMF tasks. The following section shows some experiments and results.

\subsection{Experiment and Evaluation}

The WCDOPS+NIM test is implemented in Cheddar. This section presents some experiments done to evaluate the analysis results of the test. A first experiment evaluates the WCDOPS+NIM test by simulation, while the second applies it to a real case-study from Thales. The following sections present these experiments. In each section the experimental setup is exposed, then experimental results are presented and discussed.

\subsubsection{WCDOPS+NIM Evaluation}

The WCDOPS+NIM test is compared to the original WCDOPS+ test by simulation. This experiment evaluates the pessimism of the original test when it is applied to transactions where there are non-immediate tasks. In order to compare WCDOPS+NIM with WCDOPS+, the tests are applied to randomly generated system architecture models. The models are generated according to the same parameters as the WCDOPS+ simulations in [8] so both tests can be compared. The Cheddar generator is updated for the simulations.

The generator produces system architecture models composed of 4 processors all with a preemptive FP scheduling policy. In the simulations, all processors have a utilization factor that varies between $10 \%$ to $70 \%$. A model also has 10 transactions with 10 tasks per transaction. A transaction has a period between 10 and 100000 units of time.

Initially a task has the same priority as its direct predecessor in the transaction. It is also allocated on the same processor as its direct predecessor. The direct predecessor is chosen randomly. Both priority and processor parameters can vary. The probability to choose a random priority for a task, after its default priority is set, is $0,0.25$, or 0.50 , depending on the user-defined simulation parameters. There is a probability of 0.25 to choose a random processor for a task, after its default processor is set. If a parameter varies, a random priority (resp. a random processor) is chosen for a task.

Tasks are immediate initially. When its offset is computed, a generated task can become non-immediate randomly. The probability to increase a task's offset, after its default offset is set, is 0.25 or 0.50 , depending on the user-defined simulation parameters. If a task becomes non-immediate, its offset is increased by a random value between 0 and 1000 . WCDOPS + is applied with ghost intermediate tasks added to model non-immediateness.

Simulations are conducted by making different parameters of the generator vary. For each set of parameters of the generator, 5 system architecture models 


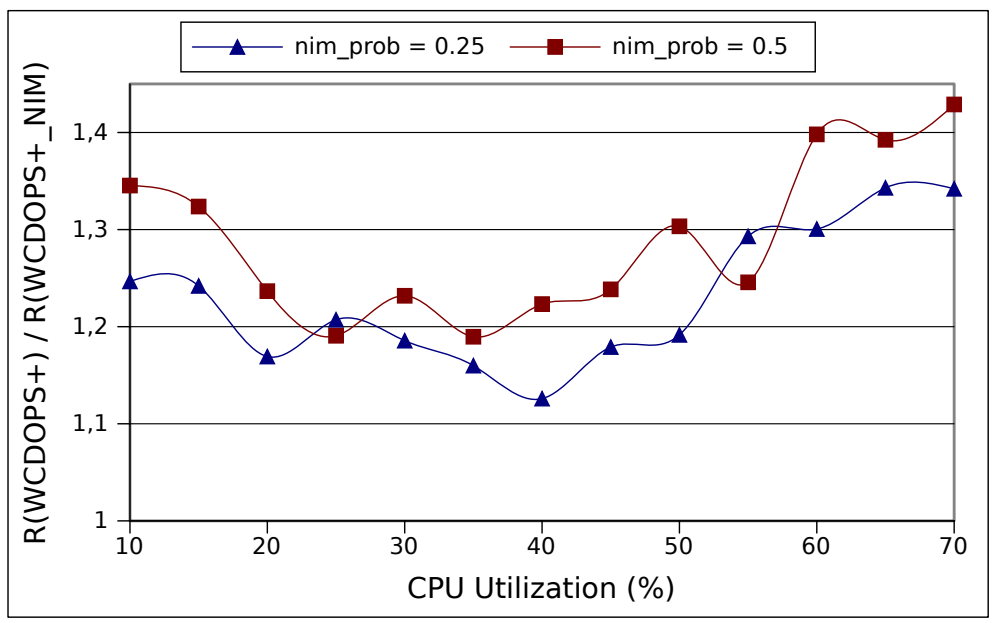

Figure 11: Comparison between WCDOPS+ and WCDOPS+NIM by Processor Utilization and Offset Increase Probability: nim_prob denotes the probability to increase a task's offset

are generated and the response times are computed for each model. For a set of generator parameters, the average ratio between WCRTs given by WCDOPS+ and WCDOPS+NIM is computed.

Figure 11 shows results of the first simulation where the processor utilization varies between $10 \%$ and $70 \%$. The probability to choose a random processor and a random priority remains at 0.25 . The evolution of the ratio is shown for a probability of 0.25 and 0.5 to increase offsets.

Figure 12 shows results of the second simulation where the processor utilization varies between $10 \%$ and $70 \%$. The probability to choose a random processor and to increase offsets remains both at 0.25 . The evolution of the ratio is shown for a probability of $0.0,0.25$ and 0.5 to choose a random priority for a task.

These simulation results show that results vary according to the processor utilization. The WCDOPS+NIM test gives less pessimistic WCRTs for lower and higher processor utilizations. The highest average ratio between a responsetime given by WCDOPS+ and WCDOPS+NIM is 1.43. Like in [8], due to the nature of the experiment, the simulation becomes unfeasible for a high processor utilization. In the experiment, the threshold is $70 \%$.

In conclusion the WCRTs computed by WCDOPS+NIM are more than $40 \%$ less than WCRTs computed by the original test, for a processor utilization of $70 \%$. Furthermore, the higher the processor utilization is, the less pessimistic the WCRTs given by WCDOPS+NIM are, compared to WCDOPS+.

\subsubsection{Experimentation on Software Radio Protocol}

As a reminder, the WCDOPS+NIM test is proposed for tree-shaped transactions that have non-immediate tasks. Such transactions may be the result of DGMF 


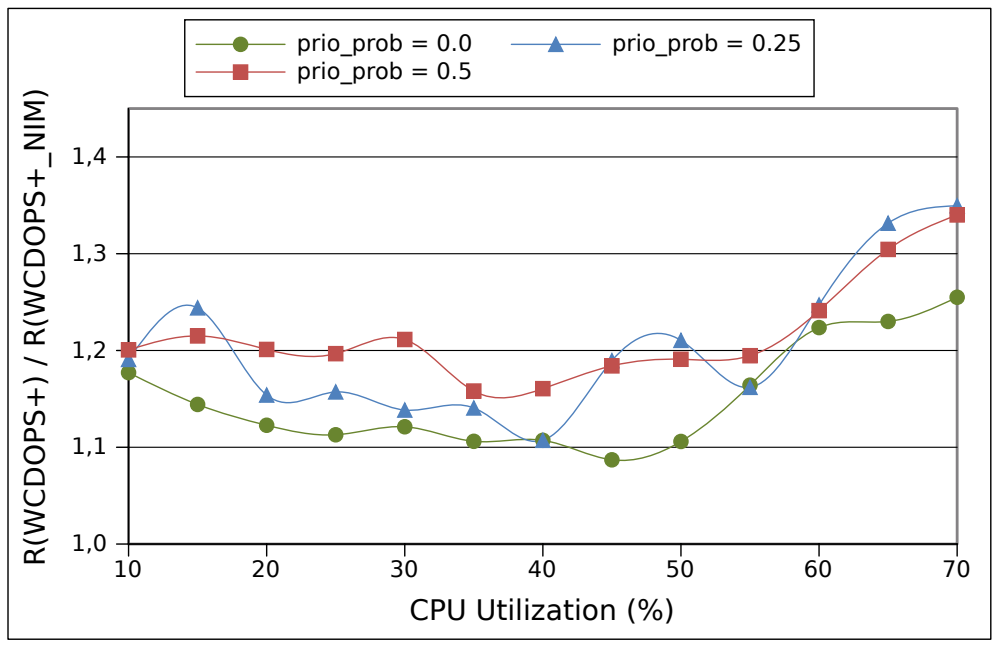

Figure 12: Comparison between WCDOPS+ and WCDOPS+NIM by Processor Utilization and Random Priority Probability: prio_prob denotes the probability to choose a random priority

transformations. The schedulability test is thus applied by first modeling the system with DGMF. To assess the gain of applying WCDOPS+NIM on a real software radio protocol, the test is compared to current practices at Thales. A case-study is used to evaluate our proposed analysis method. Besides evaluating the advantages of the proposed analysis method, this experiment also determines its scalability.

The case-study is the real software radio protocol that was presented in Section 4.3.3. As a reminder, the case-study is implemented with 8 POSIX threads on a processor called GPP1, and 4 threads on a processor called GPP2. Both processors are scheduled by the SCHED_FIFO scheduler of Linux (preemptive FP policy). The threads have precedence dependencies and they are released at the start of different slots of a TDMA frame of 14 slots. The case-study, modeled with DGMF, was transformed to a tree-shaped transaction of 44 tasks with non-immediate tasks, shown in Figure 9 of Section 4.3.3.

Currently the analysis approach used at Thales is similar to the approach of the Joseph \& Pandya test in [16] (abbreviated as the "J\&P test" in the following paragraphs). To assess the advantage of applying WCDOPS+NIM to the real software radio protocol, WCRTs given by WCDOPS+NIM are compared with those computed by the $\mathrm{J} \& \mathrm{P}$ test. The $\mathrm{J} \& \mathrm{P}$ test cannot be applied directly to the case-study for the following reasons:

- Precedence dependency between tasks that may be on different processors

- Constraint of deadline less than or equal to period, assumed by the J\&P test 
For the problem of precedence dependency between tasks on different processors, in the case-study, the priority assignment in [23] is first used when analyzing a particular task: a task has a priority lower than its predecessor's priority. All tasks are then allocated to a same processor and a synchronous system is assumed for the $J \& P$ test.

The J\&P test assumes a task deadline less than or equal to its period. Therefore the test only computes the WCRT of the first job of a task. The constraint on deadlines is not respected by the case-study tasks, so the response time of the first job is not sufficient to determine schedulability. On the other hand, as we will see with the results in the following paragraph, even the response time of the first job is overestimated by the J\&P test, compared to a WCRT computed by WCDOPS+NIM.

Only the WCRTs of tasks, without any successor, are compared. These tasks represent the completion of some service, thus their response time is of interest to determine if the service misses some deadline. For example in the service represented by the precedence dependency chain $R M_{-} S 1 \prec B B_{-} S 1_{-} 1 \prec$ $B B_{-} S 1_{-} 2 \prec B B_{-} S 1_{-} 3$, only the WCRT of $B B_{-} S 1_{-} 3$ is of interest to determine if the service misses its deadline, i.e. if the deadline of $B B_{-} S 1_{-} 3$ is missed.

When applying WCDOPS+NIM on the case-study, the convergence of response times takes 7 seconds on a Intel Core i5 @ $2.40 \mathrm{GHz}$. The computed WCRTs are shown in Table 5. From results in Table 5 , a ratio of response times, given by both tests, is computed for each task. In average, this ratio is 8.89 so in average the J\&P schedulabiltiy test gives a WCRT almost 9 times higher than WCDOPS+NIM. This result shows that considering TDMA task releases reduces the pessimism of WCRTs.

The WCDOPS + test was also applied to the case-study by modeling nonimmediateness with ghost intermediate tasks. The ratio of WCRTs computed by WCDOPS+, compared to WCRTs computed by WCDOPS+NIM, is 1.08. The pessimism of WCRTs computed by WCDOPS+ is thus negligible. This result can be explained by the fact that the processor utilization of the case-study is low since the duration of slots is high compared to the WCETs of tasks. As shown by the simulations in Section 5.3.1, for a low processor utilization, WCDOPS+ does not give significantly more pessimistic WCRTs than WCDOPS+NIM.

The slot durations are high because the initial TDMA configuration is not necessarily optimized. Furthermore some processor time must be dedicated to execution of other applications than the software radio protocol. Later specifications will decrease the slot durations, which means an increase in processor utilization.

\section{Conclusion}

\subsection{Summary}

The work presented in this article contributed to scheduling analysis of real-time radio software systems. This article focused on communication systems using 
Table 5: Task WCRTs of MAC Layer Case-Study: $R_{R M}$ is WCRT given by [16] $R_{W C D O P S+N I M}$ is WCRT (global WCRT minus offset) given by WCDOPS+NIM; Time values in ms

\begin{tabular}{|l|l|l|}
\hline Task & $R_{R M}$ & $R_{W C D O P S+N I M}$ \\
\hline FC_S1_3 & 33405 & 573 \\
\hline AB_B1_3 & 6505 & 1241 \\
\hline AB_B2_3 & 6505 & 1241 \\
\hline AB_B3_3 & 6505 & 1241 \\
\hline AB_B4_3 & 6505 & 741 \\
\hline AD_T2_1 & 3955 & 651 \\
\hline AD_T4_1 & 3955 & 651 \\
\hline AD_T6_1 & 3955 & 651 \\
\hline AD_T8_1 & 3955 & 651 \\
\hline BT_T1_1 & 1915 & 463 \\
\hline BT_T3_1 & 1915 & 463 \\
\hline BT_T5_1 & 1915 & 463 \\
\hline BT_T7_1 & 1915 & 463 \\
\hline BB_B5_3 & 16859 & 3313 \\
\hline BS_S1_3 & 16959 & 3634 \\
\hline
\end{tabular}

TDMA to access the shared communication medium. We took software radio protocols developed at Thales Communications \& Security as case-studies.

Software radio protocols have some characteristics to consider for scheduling analysis. Among these characteristics, the system has tasks released by TDMA ticks, and execution times and deadlines that depend on the TDMA slots. The tasks are also dependent, through precedence dependencies and shared resources. They execute on a partitioned multiprocessor execution platform, with preemptive fixed priority scheduling.

For scheduling analysis of a software radio protocol, we solved the issue of applicability of task models and analysis methods. Indeed, existing task models of the literature are not applicable to all characteristics of a software radio protocol.

The solution proposed in this article, is to abstract the architecture of a software radio protocol with the DGMF task model. Our task model fulfills requirements for scheduling analysis of a software radio protocol based on TDMA. Indeed, our task model expresses individual jobs of a task, called DGMF frames. DGMF extends the GMF task model with task dependencies and the proposed task model is applicable to a partitioned multiprocessor execution platform.

To analyze DGMF tasks, they are transformed to transactions. Then an adapted schedulability test, proposed in this article, is applied to the transactions. Our schedulability test is called WCDOPS+NIM, and it extends the WCDOPS+ test of the literature. The proposed test is applicable to tree-shaped transactions with non-immediate tasks.

DGMF, transactions, and their analysis methods are implemented in the 
Cheddar scheduling analysis tool. Scheduling analysis, with DGMF, can then be applied automatically.

The proposed solution was evaluated through several experiments. The modeling and transformation of DGMF tasks to transactions was evaluated both by simulation and with a real case-study. Simulation results showed that the implementation of the transformation is polynomial, when the number of DGMF frames increases, or the number of precedence dependencies increases. For a model of 100 DGMF tasks, 1000 DGMF frames, and 1100 precedence dependencies, simulation results showed that the transformation time takes about 160 ms.

DGMF was then applied for the modeling of a real case-study from Thales. This experiment showed that a real system has much less tasks, frames and precedence dependencies than the models generated in the simulation. Indeed, the case-study was modeled with 8 DGMF tasks, 43 frames and 14 precedence dependencies.

The DGMF model representing the real case-study was transformed into a transaction model. The WCDOPS+NIM test was then applied. Experimental results showed that WCDOPS+NIM computes WCRTs almost 9 times lower in average than the fundamental periodic task model test. The approach of the periodic task model analysis is used for some systems at Thales. Simulation results also showed that WCDOPS+NIM gives less pessimistic response times than WCDOPS+, as processor utilization increases. For a processor utilization of a $70 \%$, WCDOPS+NIM gives up to $40 \%$ less pessimistic WCRTs.

In conclusion the proposed solution solves the issues faced by scheduling analysis of a software radio protocol based on TDMA. Experimental results also show that the solution is scalable to systems developed at Thales Communications \& Security.

\subsection{Future Works}

In the future, we wish to improve the DGMF analysis method, by extending the WCDOPS+NIM schedulability test. The analysis method assumes that DGMF tasks respect the Cycle Separation property. This means that the deadline of job $p$ of $F_{i}^{N_{i}}$ is less than the release of job $p+1$ of $F_{i}^{1}$. By assuming this property, the first frame $F_{i}^{1}$ should not interfere the last frame $F_{i}^{N_{i}}$, unless the last frame misses a deadline. To analyze DGMF tasks that do not respect the Cycle Separation property, the WCDOPS+NIM schedulability test needs to consider this behavior.

The WCDOPS+NIM test is currently applicable to tree-shaped transactions. There exists an extension of WCDOPS + for graph-shaped transactions in [37. In the future, the WCDOPS+NIM test can be adapted for graph-shaped transactions with non-immediate tasks.

Works can also be done on exploiting the DGMF task model with higher level architecture models. In [34, we proposed an experimental architecture model of a software radio protocol in UML MARTE [38, that can be used for schedulability analysis with DGMF but The MARTE model and its transformation to 
DGMF, have to be formalized in the future.

Finally, we should also investigate how DGMF can exploit other architecture models described with languages such as AADL 39, and EAST-ADL 40. This would also investigate furthermore the applicability of DGMF to domains such as avionic and automotive.

\section{References}

[1] J. Mitola, The software radio architecture, IEEE Communications Magazine 33 (5) (1995) 26-38.

[2] T. Chan, Time-division multiple access, in: Handbook of Computer Networks, John Wiley \& Sons, Hoboken, 2011, pp. 769-778.

[3] C. Liu, J. Layland, Scheduling algorithms for multiprogramming in a hardreal-time environment, Journal of the ACM 20 (1) (Jan, 1973) 46-61.

[4] L. Sha, T. Abdelzaher, K.-E. Arzen, A. Cervin, T. Baker, A. Burns, G. Buttazzo, M. Caccamo, J. Lehoczky, A. K. Mok, Real time scheduling theory: A historical perspective, Real-Time Systems 28 (2-3) (Nov-Dec, 2004) 101155.

[5] S. Baruah, Dynamic- and static-priority scheduling of recurring real-time tasks, Real-Time Systems 24 (1) (Jan, 2003) 93-128.

[6] S. Baruah, D. Chen, S. Gorinsky, A. Mok, Generalized multiframe tasks, Real-Time Systems 17 (1) (Jul, 1999) 5-22.

[7] K. Tindell, J. Clark, Holistic schedulability analysis for distributed hard real-time systems, Microprocessing and Microprogramming 40 (2-3) (Apr, 1994) 117-134.

[8] O. Redell, Analysis of tree-shaped transactions in distributed real time systems, in: Proceedings of the 16th Euromicro Conference on Real-Time Systems, Catania, Italy, 2004, pp. 239-248.

[9] F. Singhoff, A. Plantec, P. Dissaux, J. Legrand, Investigating the usability of real-time scheduling theory with the cheddar project, Real-Time Systems 43 (3) (Jun, 2009) 259-295.

[10] H. Zimmermann, OSI reference model-the ISO model of architecture for open systems interconnection, IEEE Transactions on Communications 28 (4) (1980) 425-432.

[11] N. Malcolm, W. Zhao, The timed-token protocol for real-time communications, Computer 27 (1) (Jan, 1994) 35-41.

[12] R. Rajkumar, Real-time synchronization protocols for shared memory multiprocessors, in: Proceedings of the 10th International Conference on Distributed Computing Systems, Paris, France, 1990. 
[13] L. Sha, R. Rajkumar, J. Lehoczky, Priority inheritance protocols: an approach to real-time synchronization, IEEE Transactions on Computers 39 (9) (1990) 1175-1185.

[14] H. Kopetz, Event-triggered versus time-triggered real-time systems, in: Operating Systems of the 90s and Beyond, Vol. 563 of Lecture Notes in Computer Science, Springer Berlin Heidelberg, 1991, pp. 86-101.

[15] H. Kopetz, The time-triggered model of computation, in: Proceedings of the 19th IEEE Real-Time Systems Symposium, Madrid, Spain, 1998.

[16] M. Joseph, P. Pandya, Finding response times in a real-time system, The Computer Journal 29 (5) (1986) 390-395.

[17] J. Palencia, M. Harbour, Exploiting precedence relations in the schedulability analysis of distributed real-time systems, in: Proceedings of the 20th IEEE Real-Time Systems Symposium, Phoenix, AZ, 1999, pp. 328-339.

[18] E. Wandeler, L. Thiele, Optimal TDMA time slot and cycle length allocation for hard real-time systems, in: Proceedings of the 11th Asia and South Pacific Design Automation Conference, Yokohama, Japan, 2006.

[19] S. Baruah, V. Bonifaci, A. Marchetti-Spaccamela, L. Stougie, A. Wiese, A generalized parallel task model for recurrent real-time processes, in: Proceedings of the 33rd Real-Time Systems Symposium, San Juan, Puerto Rico, 2012.

[20] H. Takada, K. Sakamura, Schedulability of generalized multiframe task sets under static priority assignment, in: Proceedings of the 4th International Workshop on Real-Time Computing Systems and Applications, Taipei, Taiwan, 1997, pp. 80-86.

[21] J. Palencia, M. Harbour, Schedulability analysis for tasks with static and dynamic offsets, in: Proceedings of the 19th IEEE Real-Time Systems Symposium, Madrid, Spain, 1998.

[22] H. Chetto, M. Silly, T. Bouchentouf, Dynamic scheduling of real-time tasks under precedence constraints, Real-Time Systems 2 (3) (1990) 181-194.

[23] N. Audsley, K. Tindell, A. Burns, The end of the line for static cyclic scheduling?, in: Proceedings of the 5th Euromicro Workshop on Real-Time Systems, Oulu, Finland, 1993.

[24] J. Forget, F. Boniol, E. Grolleau, D. Lesens, C. Pagetti, Scheduling dependent periodic tasks without synchronization mechanisms, in: Proceedings of the 16th IEEE Real-Time and Embedded Technology and Applications Symposium, Stockholm, Sweden, 2010. 
[25] M. Forget, E. Grolleau, C. Pagetti, P. Richard, Dynamic priority scheduling of periodic tasks with extended precedences, in: Proceedings of the 16th Conference on Emerging Technologies \& Factory Automation, Toulouse, France, 2011.

[26] V. Bonifaci, A. Marchetti-Spaccamela, S. Stiller, A. Wiese, Feasibility analysis in the sporadic DAG task model, in: Proceedings of the 25th Euromicro Conference on Real-Time Systems, Paris, France, 2013.

[27] P. Ekberg, N. Guan, M. Stigge, W. Yi, An optimal resource sharing protocol for generalized multiframe tasks, in: Nordic Workshop on Programming Theory, Vasteras, Sweden, 2011.

[28] A. Rahni, Contributions à la validation d'ordonnancement temps réel en présence de transactions sous priorités fixes et EDF, Ph.D. thesis, Université de Poitiers, Poitiers, France (2008).

[29] K. Tindell, Adding time-offsets to schedulability analysis, Tech. rep., University of York (1994).

[30] S. Klaus, Finding a minimal transitive reduction in a strongly conncected digraph within linear time., in: Proceedings of the 15th International Workshop on Graph-Theoretic Concepts in Computer Science, Vol. 411, Berlin, Germany, 1990.

[31] A. Choquet-Geniet, E. Grolleau, Minimal schedulability interval for realtime systems of periodic tasks with offsets, Theoretical Computer Science 310 (Jan, 2004) 117-134.

[32] A. Burns, A. Wellings, Real-time systems and programming languages: Ada 95, real-time Java, and real-time POSIX, Pearson Education, 2001.

[33] S. Li, F. Singhoff, S. Rubini, M. Bourdellès, Extending schedulability tests of tree-shaped transactions for TDMA radio protocols, in: Proceedings of the 19th IEEE International Conference on Emerging Technology \& Factory Automation, Barcelona, Spain, 2014.

[34] S. Li, Scheduling analysis of tasks constrained by time-division multiplexing: application to software radio protocols, Ph.D. thesis, Universit de Bretagne Occidentale, Brest, France (Nov, 2014).

[35] J. Garcia, J. Gutierrez, M. Harbour, Schedulability analysis of distributed hard real-time systems with multiple-event synchronization, in: Proceedings of the 12th Euromicro Conference on Real-Time Systems, Stockholm, Sweden, 2000.

[36] J. Lehoczky, Fixed priority scheduling of periodic task sets with arbitrary deadlines, in: Procedings of the 11th Real-Time Systems Symposium, Lake Buena Vista, USA, 1990, pp. 201-209. 
[37] J. Kany, S. Madsen, Design optimisation of fault-tolerant event-triggered embedded systems, Master's thesis, Technical University of Denmark, Lyngby, Denmark (2007).

[38] M. Z. Iqbal, S. Ali, T. Yue, L. Briand, Applying UML/MARTE on industrial projects: challenges, experiences, and guidelines, Software \& Systems Modeling (Mar, 2014) 1-19.

[39] P. H. Feiler, D. P. Gluch, J. J. Hudak, The architecture analysis \& design language (AADL): an introduction, Tech. rep., Software Engineering Institute, Pittsburgh (2006).

[40] V. Debruyne, F. Simonot-Lion, Y. Trinquet, EAST-ADL - an architecture description language, in: Architecture Description Languages, Vol. 176, Springer-Verlag, New York, USA, 2005, pp. 181-195. 